\title{
Production of secondary particles and nuclei in cosmic rays collisions with the interstellar gas using the FLUKA code
}

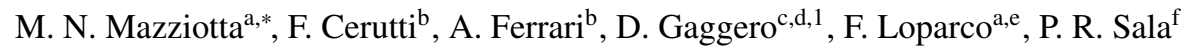 \\ ${ }^{a}$ Istituto Nazionale di Fisica Nucleare, Sezione di Bari, 70126 Bari, Italy \\ ${ }^{b}$ CERN, Geneva, Switzerland \\ ${ }^{c}$ SISSA, via Bonomea 265, 34136 Trieste, Italy \\ ${ }^{d}$ INFN, Sezione di Trieste, via Valerio 2, 34127 Trieste, Italy \\ e Dipartimento di Fisica "M. Merlin" dell'Università e del Politecnico di Bari, I-70126 Bari, Italy \\ ${ }^{f}$ Istituto Nazionale di Fisica Nucleare, Sezione di Milano, 20133 Milano, Italy
}

\section{Abstract}

The measured fluxes of secondary particles produced by the interactions of Cosmic Rays (CRs) with the astronomical environment play a crucial role in understanding the physics of CR transport. In this work we present a comprehensive calculation of the secondary hadron, lepton, gamma-ray and neutrino yields produced by the inelastic interactions between several species of stable or long-lived cosmic rays projectiles $\left(\mathrm{p}, \mathrm{D}, \mathrm{T},{ }^{3} \mathrm{He},{ }^{4} \mathrm{He},{ }^{6} \mathrm{Li},{ }^{7} \mathrm{Li},{ }^{9} \mathrm{Be},{ }^{10} \mathrm{Be},{ }^{10} \mathrm{~B},{ }^{11} \mathrm{~B},{ }^{12} \mathrm{C},{ }^{13} \mathrm{C},{ }^{14} \mathrm{C},{ }^{14} \mathrm{~N},{ }^{15} \mathrm{~N},{ }^{16} \mathrm{O},{ }^{17} \mathrm{O},{ }^{18} \mathrm{O}\right.$, ${ }^{20} \mathrm{Ne},{ }^{24} \mathrm{Mg}$ and $\left.{ }^{28} \mathrm{Si}\right)$ and different target gas nuclei $\left(p,{ }^{4} \mathrm{He},{ }^{12} \mathrm{C},{ }^{14} \mathrm{~N},{ }^{16} \mathrm{O},{ }^{20} \mathrm{Ne},{ }^{24} \mathrm{Mg},{ }^{28} \mathrm{Si}\right.$ and $\left.{ }^{40} \mathrm{Ar}\right)$. The yields are calculated using FLUKA, a simulation package designed to compute the energy distributions of secondary products with large accuracy in a wide energy range. The present results provide, for the first time, a complete and self-consistent set of all the relevant inclusive cross sections regarding the whole spectrum of secondary products in nuclear collisions. We cover, for the projectiles, a kinetic energy range extending from $0.1 \mathrm{GeV} / \mathrm{n}$ up to $100 \mathrm{TeV} / \mathrm{n}$ in the lab frame. In order to show the importance of our results for multi-messenger studies about the physics of CR propagation, we evaluate the propagated spectra of Galactic secondary nuclei, leptons, and gamma rays produced by the interactions of CRs with the insterstellar gas, exploiting the numerical codes DRAGON and GammaSky. We show that, adopting our cross section database, we are able to provide a good fit of a complete sample of CR observables, including: leptonic and hadronic spectra measured at Earth, the local interstellar spectra measured by Voyager, and the gamma-ray emissivities from Fermi-LAT collaboration. We also show a set of gamma-ray and neutrino full-sky maps and spectra.

Keywords: Cosmic Ray propagation, Collisions with interstellar medium, Secondary particle production

\section{Introduction}

According to the currently accepted scenario, Galactic Cosmic Rays (CRs) are accelerated in Supernova Remnants (SNRs) and interact with the turbulent interstellar magnetic field: the resulting motion is well described by a diffusion equation. During their journey through the Galaxy, the inelastic collisions of hadronic CRs with the interstellar medium (ISM), produce lighter particles and secondary radiation: the study of these events is very important since it may shed light on the origin of the CRs themselves and on the mechanisms governing their transport.

For example, the production of light nuclei - such as Boron from heavier ones (in particular Carbon and Nitrogen) has been extensively studied in recent times, since light nuclei ratios (e.g. $\mathrm{B} / \mathrm{C}, \mathrm{N} / \mathrm{O}$ ) are often used to constrain the propagation models in the Galaxy and in particular the rigidity dependence and normalization of the diffusion coefficient (see for instance [1, [2, 3]).

\footnotetext{
${ }^{*}$ Corresponding author

Email address: mazziotta@ba.infn.it (M. N. Mazziotta)

${ }^{1}$ Current address: GRAPPA Institute, University of Amsterdam, Science Park 904, 1090 GL Amsterdam, The Netherlands
}

Moreover, the collisions of protons and Helium nuclei with the gas, and subsequent decays of the produced neutral pions $\left(\pi^{0} \mathrm{~s}\right)$, are expected to give the most relevant contribution to the gamma-ray diffuse emission in the Galactic plane, since that is the region with the largest gas column densities. In this context, the high precision gamma-ray maps and spectra measured by the Fermi-LAT instrument in the energy band spanning from several tens of $\mathrm{MeV}$ to several hundreds of $\mathrm{GeV}$ [4], as well as the data in the $\mathrm{TeV}$ range from ground Air Cerenkov Telescopes [5, 7, 6, 8], are very useful and represent a unique opportunity to understand the CR transport properties in different regions of the Galaxy [9, 10, 11].

Concerning both the study of gamma-ray point sources and the diffuse emission, the radiation in the $\mathrm{MeV}-\mathrm{TeV}$ energy range can also be produced by electron bremsstrahlung and inverse Compton (IC) scattering. The relative contributions of leptonic and hadronic processes depend on many parameters (i.e. the environment around the source), often resulting into considerable uncertainties in the physical interpretation of the observed gamma-ray emission. For this reason, an accurate knowledge of the gamma-ray spectra resulting from hadronic interactions is crucial for the physical interpretation of the 


\section{emission phenomena.}

Gamma rays are also produced in the interactions of cosmic-ray nuclei with the Earth's atmosphere. The Fermi collaboration recently inferred the proton spectrum from a measurement of the spectrum of gamma rays originated from the Earth limb (i.e. the top of the atmosphere) [12]. This measurement allowed the reconstruction of the CR proton spectrum in an energy range from $90 \mathrm{GeV}$ up to $6 \mathrm{TeV}$. The study of the CR spectra in this region is a topic of extreme scientific interest, since the PAMELA collaboration reported a hardering of the proton and helium spectra around $200 \mathrm{GeV}[13$ ] that has been recently confirmed by the AMS02 collaboration [14, 15]. Clearly, also for such analysis, the precise knowledge of the gamma-ray yields produced in the hadronic interactions of CRs with atmospheric nuclei is very crucial.

Charged leptons and antiprotons are another important product of the inelastic hadronic collisions of CRs with the interstellar gas. The PAMELA collaboration measured the positron, electron and antiproton spectra, as well as the spectra of many light nuclei [16, 17, 18, 19, 13, 20, 21]. Recently the AMS02 collaboration provided very accurate results on the positron fraction (i.e. the ratio $\left.e^{+} /\left(e^{-}+e^{+}\right)\right)$[22, 23] and new high-precision results on the positron and electron intensities [24]. They also presented preliminary results on the $\mathrm{B} / \mathrm{C}$ ratio $[25,26]$. All those datasets were taken during the same period, and this circumstance makes the interpretation of the data easier. Simultaneous measurements of several particle spectra performed by the same experiment over a wide energy range will in fact ensure reduced experimental systematics and will also limit the uncertainties arising from the $\mathrm{CR}$ propagation in the heliosphere (solar modulation) and in the Galaxy.

Since the hadronic interactions (in particular the $p-$ $p$ interaction) are the main processes responsible of the production of secondary particles such as gamma rays, neutrinos, electrons and positrons, and since the decays of pions and kaons play a major role in these processes, several parameterizations were developed over the years to describe the production of these mesons in $p-p$ interactions (see for instance [27, 28, 29]). These calculations are mainly based on the inclusive cross section of pion production in $p-p$ collisions, evaluated from the accelerator data. However, the models may lose accuracy in the high-energy region, where experimental data have established a logarithmic increase of the total inelastic cross section with the incident proton energy [30].

In addition, the contribution of the gamma-ray production from the decays of other particles than neutral pions was also found to be not negligible. Finally, for low-energy primaries $(<10 \mathrm{GeV})$, the contribution of resonances to the secondary production cannot be neglected.

In addition to the theoretical methods, many parameterizations based on Monte Carlo (MC) calculations were proposed [31, 32, 33, 34, 35, 36]. These calculations were performed using event generators developed for the high energy particle physics (e.g. PYTHIA [37], DPMJET-III [38], SYBILL [39], QGSJET-II [40]). The main advantage of the MC approach is that the codes can be used directly to calculate the spectra of all secondary species. The MC approach also allows a direct evaluation of the secondary particle yields in nucleusnucleus interactions, instead of using parameterizations and/or scaling factors based only on the $p-p$ interactions (see for instance [35]). However, the event generators included in most $\mathrm{MC}$ codes are reliable only above a few $10 \mathrm{GeV} / \mathrm{n}$, and parameterizations need to be implemented to describe the low energy region [31, 33, 36].

A common practice in the literature is to fit different observables (e.g. hadronic and leptonic spectra) using cross sections obtained from several parameterizations, derived with different methods and under different assumpions: this may lead to inconsistencies in the determination of CR diffusion models. In the present work we aim at providing a complete and consistent set of cross sections for the secondary production of hadrons and leptons. We perform our study with the FLUKA MC simulation code [41, 42, 43, 46].

The paper is organized as follows: first we describe the main features of FLUKA, then we present and discuss the main results regarding the secondary production cross sections for the interactions between several species of CR projectiles and different target nuclei. Finally, we discuss the impact of our results on CR propagation models, using a custom version of the propagation code DRAGON. In particular, we present a set of three models - adopting the new cross sections - tuned on a complete set of CR observables in a wide energy range.

\section{Monte Carlo simulations with FLUKA}

FLUKA is a general purpose MC code for the simulation of hadronic and electromagnetic interactions. It is used in many applications, and is continuously checked using the available data from low energy nuclear physics, highenergy accelerator experiments and measurements of particle fluxes in the atmosphere. Hadronic interactions are treated in FLUKA following a theory-driven approach. The general phenomenology is obtained from a microscopical description of the interactions between the fundamental constituents, appropriate for the different energy regions. Below a few $\mathrm{GeV}$, hadron-nucleon interaction model is based on resonance production and decay of particles, while for higher energies the Dual Parton Model (DPM) is used, implying a treatment in terms of quark chain formation and hadronization. The extension from hadron-nucleon to hadron-nucleus interactions is done in the framework of the PreEquilibrium Approach to NUclear Thermalization model (PEANUT) [47, 48], including the Gribov-Glauber multi-collision mechanism followed by the pre-equilibrium stage and eventually equilibrium processes (evaporation, fission, Fermi break-up and gamma deexcitation). The models used in FLUKA are benchmarked against the available data from experiments. More details about the FLUKA package can be found in the manual [41, 42] and a description of hadronic interactions model used in FLUKA can be found in Ref. [49].

FLUKA can simulate with high accuracy the interaction and propagation in matter of about 60 different species of particles, including photons and electrons from $1 \mathrm{keV}$ to thousands of 


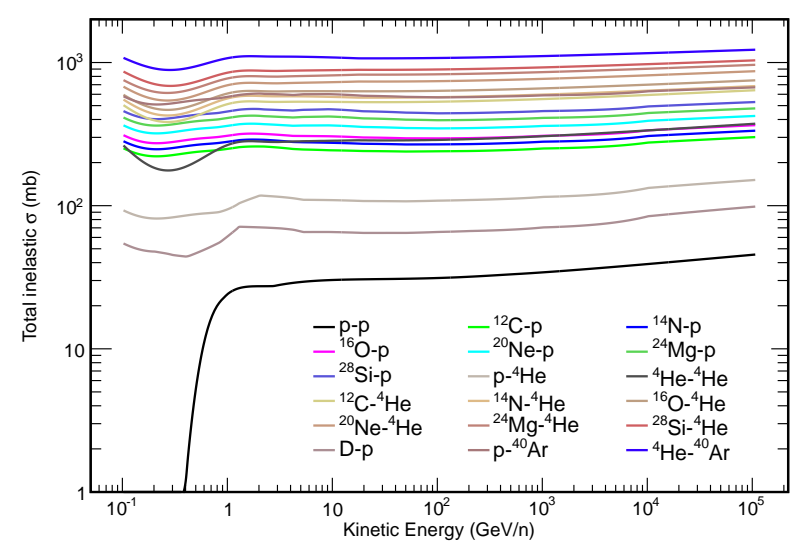

Figure 1: Total inelastic cross sections as a function of the energy per nucleon of the incoming projectile. The plot shows the cross sections for all the projectiletarget pairs studied in the present work.

$\mathrm{TeV}$, neutrinos, muons of any energy, hadrons of energies up to $20 \mathrm{TeV}$ (up to $10 \mathrm{PeV}$ when it is interfaced with the DPMJET code [38]) and all the corresponding antiparticles, neutrons down to thermal energies and heavy ions.

In addition, FLUKA can handle even very complex geometries, using an improved version of the well known Combinatorial Geometry (CG) package. The FLUKA CG has been designed to track correctly also charged particles, even in the presence of magnetic fields. The predictions of FLUKA have been checked with a large set of experimental data collected in accelerator experiments. Few examples of applications in CR physics can be found in [50, 51, 52].

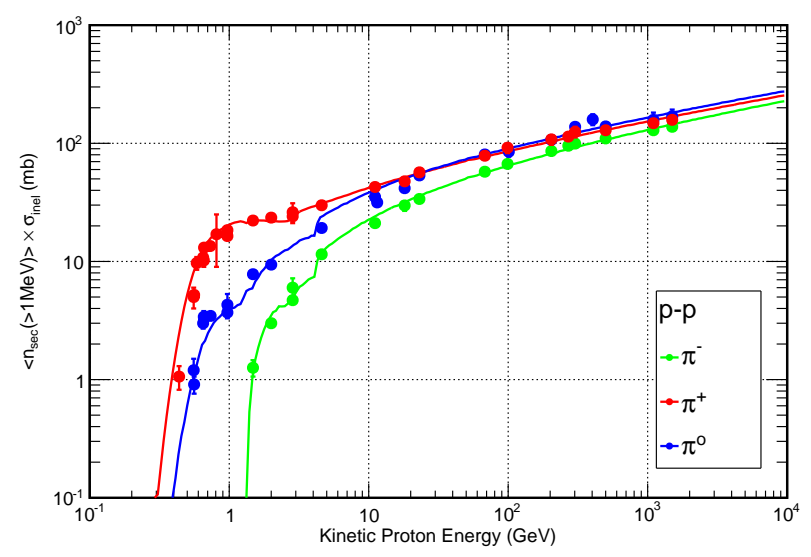

Figure 2: Inclusive cross sections for the production of $\pi^{0}$ (blue), $\pi^{+}$(red) and $\pi^{-}$(green) in $p-p$ collision as function of the incoming proton kinetic energy. Lines: FLUKA simulation; points: data from Ref. [28].

\section{Secondary particle production in $\mathrm{CR}$ interactions}

In this work we use a developement version of FLUKA [43, 44, 45, 46] to evaluate the yields of secondary particles and spallation nuclei in CR interactions. In case of nucleusnucleus interactions DPMJET-III and a modified version [55] of RQMD [56, 57, 58] are used as external event generators. Both generators have been satisfactorily benchmarked in their respective range of application [59,60].

Our focus is on the interactions of CRs with the ISM and with the Earth's atmosphere. As it is well known, the ISM consists of $90 \%$ Hydrogen, $9.9 \%$ Helium and $0.1 \%$ atoms of heavier elements synthesized in stellar interiors [53, 54], while the atmosphere is composed of $78 \%$ of $\mathrm{N}_{2}, 21 \%$ of $\mathrm{O}_{2}$ and small fractions of other gases (mainly Argon). Primary CRs, on the other hand, are accelerated in astrophysical sources and their hadronic component consists of protons, Helium, and other heavier nuclei [30]. Therefore, we simulate the interactions of the following stable and long lived CR projectiles: $p, D, T$, ${ }^{3} \mathrm{He},{ }^{4} \mathrm{He},{ }^{6} \mathrm{Li},{ }^{7} \mathrm{Li},{ }^{9} \mathrm{Be},{ }^{10} \mathrm{Be},{ }^{10} \mathrm{~B},{ }^{11} \mathrm{~B},{ }^{12} \mathrm{C},{ }^{13} \mathrm{C},{ }^{14} \mathrm{C},{ }^{14} \mathrm{~N},{ }^{15} \mathrm{~N}$, ${ }^{16} \mathrm{O},{ }^{17} \mathrm{O},{ }^{18} \mathrm{O},{ }^{20} \mathrm{Ne},{ }^{24} \mathrm{Mg}$, and ${ }^{28} \mathrm{Si}$ impinging on $\mathrm{p},{ }^{4} \mathrm{He},{ }^{12} \mathrm{C}$, ${ }^{14} \mathrm{~N},{ }^{16} \mathrm{O}{ }^{20} \mathrm{Ne},{ }^{24} \mathrm{Mg},{ }^{28} \mathrm{Si}$, and ${ }^{40} \mathrm{Ar}$. For each interaction we simulate a sample of events, each corresponding to a value of the projectile kinetic energy $T$ (the number of simulated events is $10^{5}$ for each input energy value). The values of $T$ are taken from a grid of 285 values equally spaced on a logarithmic scale, ranging from $0.1 \mathrm{GeV} / \mathrm{n}$ up to $10^{5} \mathrm{GeV} / \mathrm{n}$.

The goal of our study is to evaluate, for each interaction process, the set of differential inclusive cross sections $d \sigma\left(T_{s} \mid T\right) / d T_{s}$, where $T_{s}$ is the kinetic energy of the given secondary and $T$ is the kinetic energy of the projectile. The differential cross section can be expressed as:

$$
\frac{d \sigma\left(T_{s} \mid T\right)}{d T_{s}}=\sigma_{\text {inel }}(T) \times \frac{d n\left(T_{s} \mid T\right)}{d T_{s}}
$$

where $\sigma_{\text {inel }}(T)$ is the total inelastic cross section and $d n\left(T_{s} \mid T\right) / d T_{s}$ is the differential multiplicity spectrum of the secondary particle to be studied.

Since we are interested in the production of stable particles, after each collision all the secondary products are followed inside a spherical volume filled with vacuum with a very large

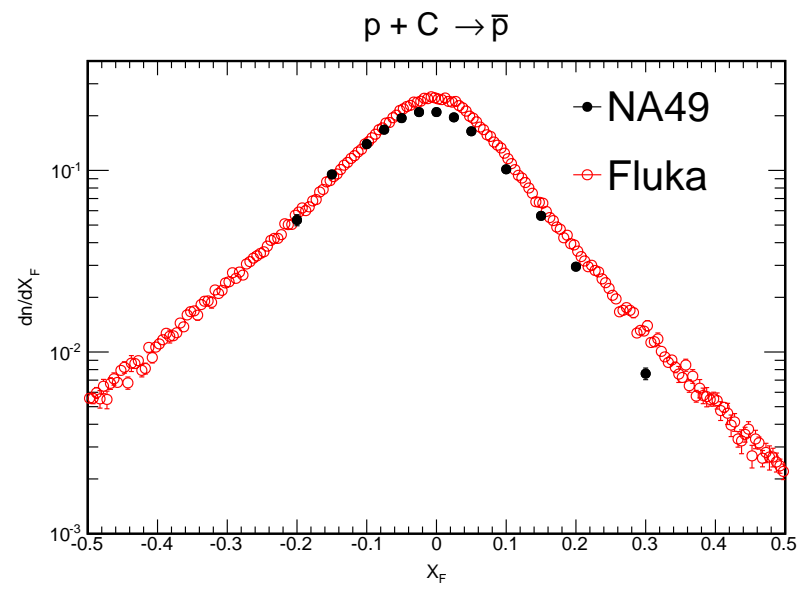

Figure 3: Feynman $\mathrm{X}\left(X_{F}\right)$ distribution for $\bar{p}$ production in proton interactions on carbon at $158 \mathrm{GeV} / \mathrm{c}$ beam momentum. The NA49 data [64] (black points) are compared with the predictions by FLUKA (red points). 

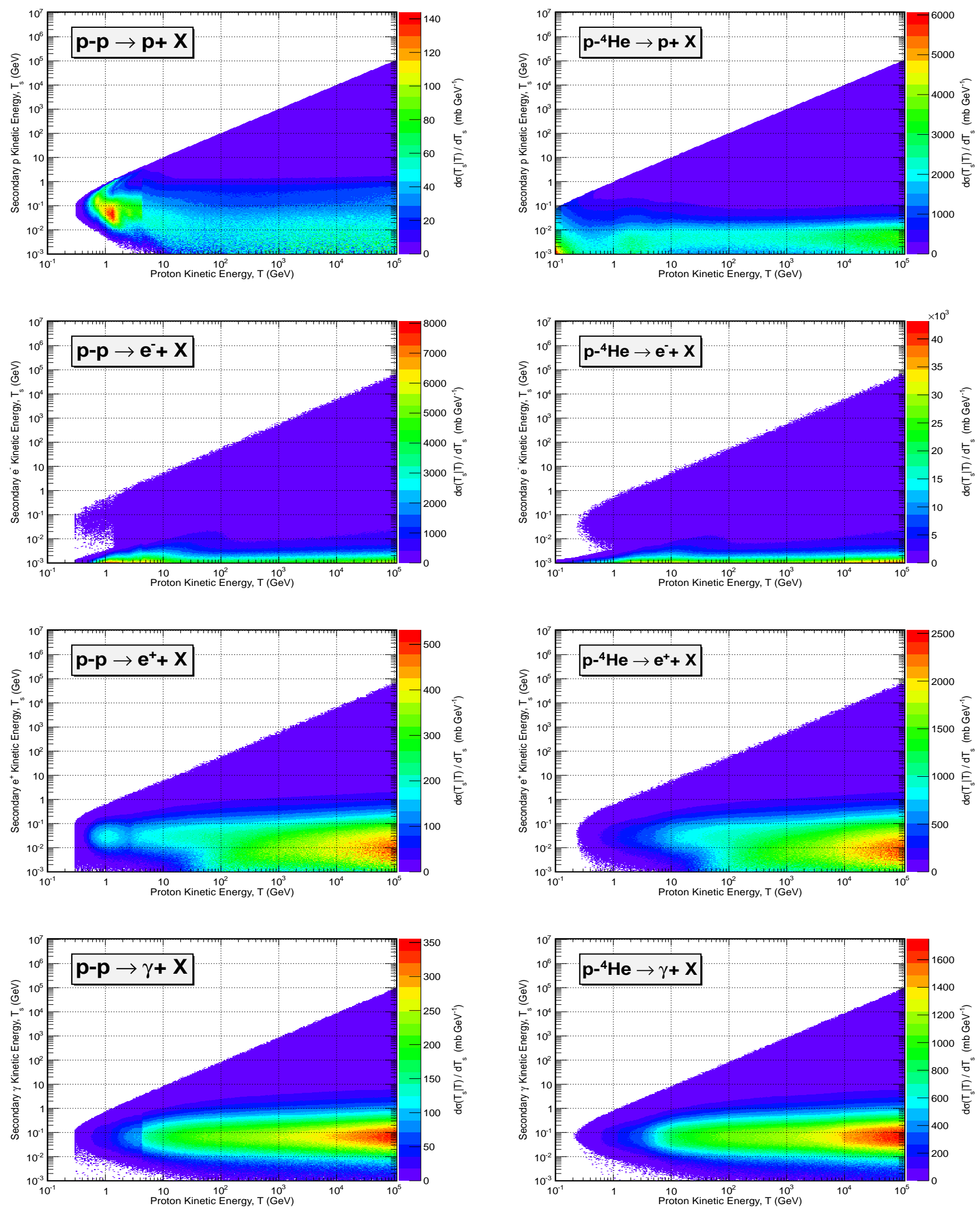

Figure 4: Differential inclusive secondary cross sections for the production of $p, e^{-}, e^{+}$and $\gamma$ in $p-p$ (left) and $p-{ }^{4} H e$ (right) interactions. The values of the cross sections (color scales) are in $\mathrm{mb} \mathrm{GeV}^{-1}$. 

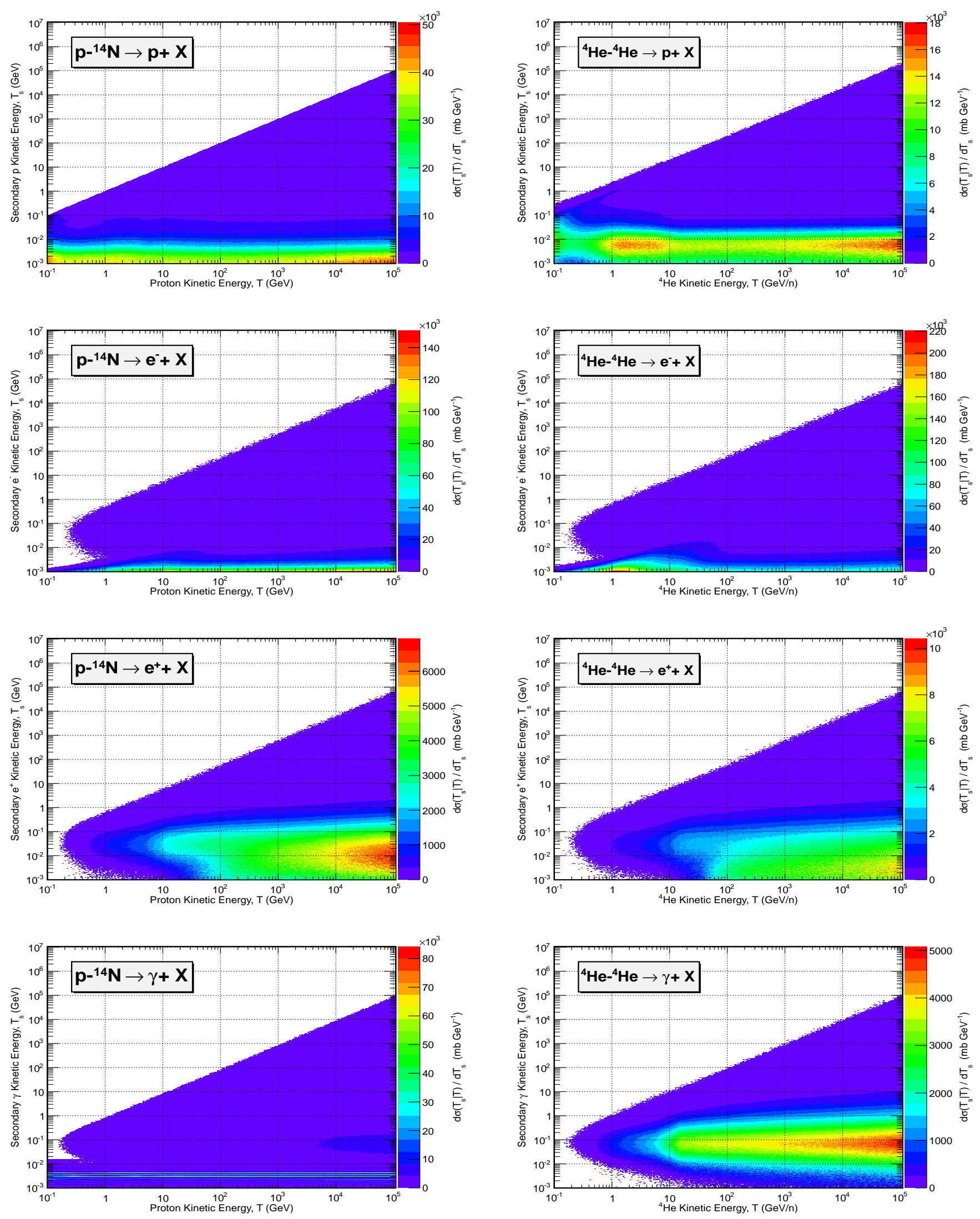

Figure 5: Differential inclusive secondary cross sections for the production of $p, e^{-}, e^{+}$and $\gamma$ in $p-{ }^{14} \mathrm{~N}$ (left) and ${ }^{4} \mathrm{He}-{ }^{4} \mathrm{He}$ (right) interactions. The values of the cross sections (color scales) are in $\mathrm{mb} \mathrm{GeV}^{-1}$. 

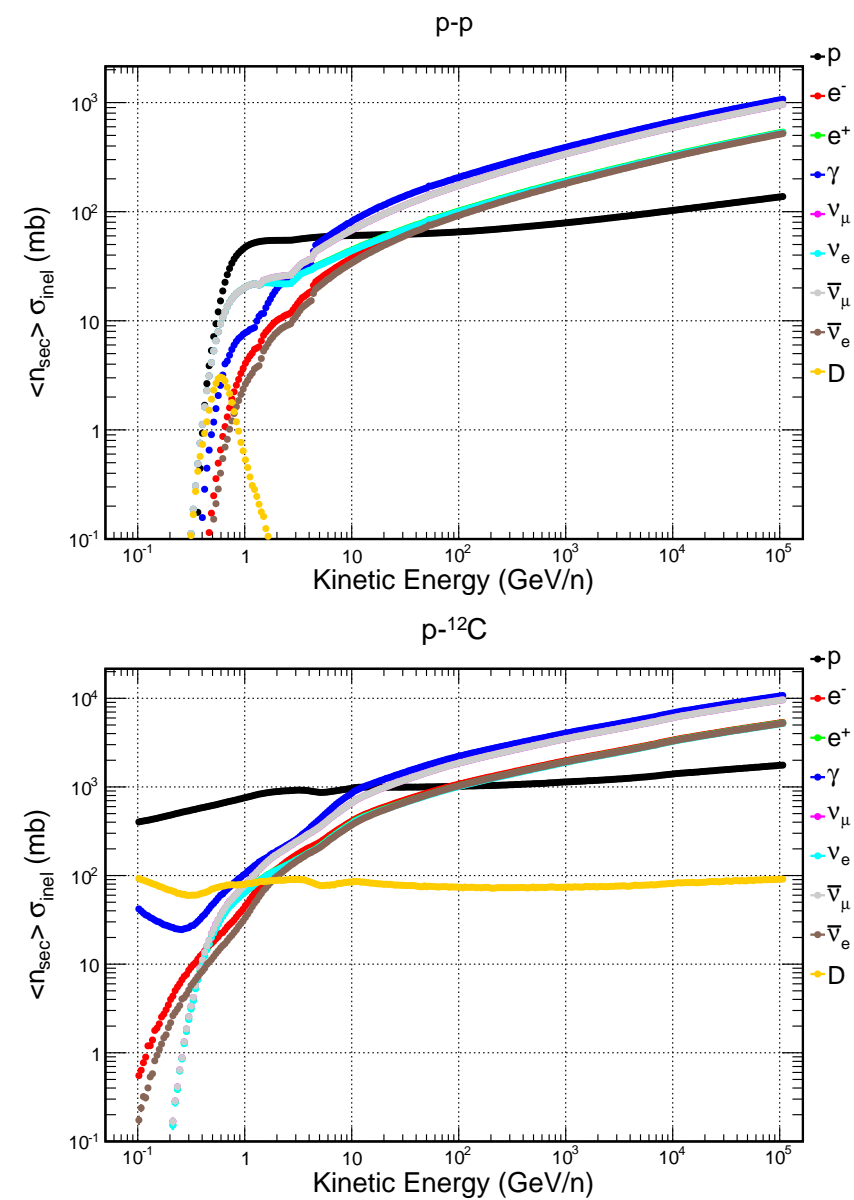
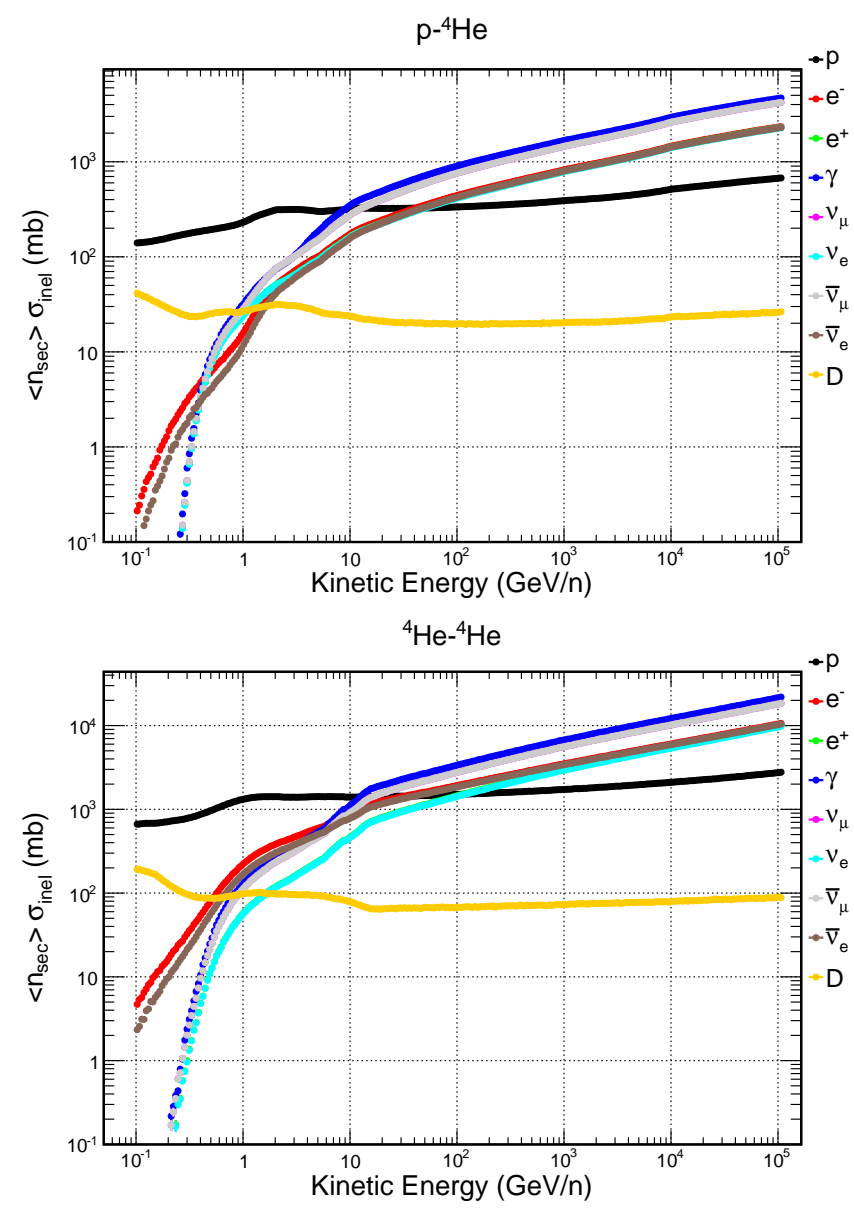

Figure 6: Inclusive cross sections for the production of protons (black), electrons (red), positrons (green), gamma rays (blue), electron neutrinos (cyan), electron antineutrinos (grey), muon neutrinos (magenta), muon antineutrinos (brown) and Deuterons (orange) in the collisions of $p-p, p-{ }^{4} \mathrm{He}, p-{ }^{12} \mathrm{C}$ and ${ }^{4} \mathrm{He}-{ }^{4} \mathrm{He}$.

radius to allow the decay of all unstable particles apart the radionuclides within the volume of the sphere.

The inclusive cross sections calculated in this way will rule the $\mathrm{CR}$ propagation within the astronomical environment. All stable secondaries produced in each interaction will be propagated together with the primaries and will possibly undergo further interactions according to the corresponding cross sections. In a similar way, unstable nuclei produced in spallation processes will be able either to interact or decay according to their cross sections and lifetimes.

The simulation with FLUKA is performed with the following settings:

- the PEANUT package is activated in the whole energy range for any reaction involving a proton as projectile or target;

- the minimum kinetic energy for DPMJET-III is set to $10 \mathrm{GeV} / \mathrm{n}$ (applying only to reactions between two nuclei heavier than a proton);

- the minimum kinetic energy for RQMD is set to $0.100 \mathrm{GeV} / \mathrm{n}$ (applying only to reactions between two nuclei heavier than a proton).

The first quantities we want to investigate are the total inelastic cross sections for the collisions we are focusing on, and - as an important cross check - the pion production cross section (for the proton-proton case) compared to current data.

Fig. 11 shows the total inelastic cross section calculated in the FLUKA code for the collision processes investigated in the present work. Our cross section model was validated with the most recent LHC data, as shown in Fig. 4 of Ref. [61].

Fig. 2 shows the pion inclusive cross sections in $p-$ $p$ collisions calculated from the FLUKA simulation at the interaction level compared to current data. It is worth here to point out that some short-lived particles produced in the interaction (i.e. $\eta$ ) promptly decay in this phase, and the decayed products cannot be separated (i.e. $\eta \rightarrow \pi$ ). Therefore the calculated inclusive cross sections shown in Fig. 2 include these products.

As mentioned above, the FLUKA results are compared against the available data from experiments: the results of these comparisons are already available in the papers cited in the current work. In particular, in Ref. [43] the double differential $\pi^{+}$production cross sections for $158 \mathrm{GeV} / \mathrm{c}$ protons on carbon as a function of transverse momentum $p_{T}$ for different values of the Feynman $X$ are compared with experimental data [62]. In the same reference, a comparison of the $p_{T}$ integrated yields of pions (for $158 \mathrm{GeV} / \mathrm{c}$ protons on carbon [62]) and kaons (for $158 \mathrm{GeV} / \mathrm{c}$ protons on proton [63]) with the data is also 

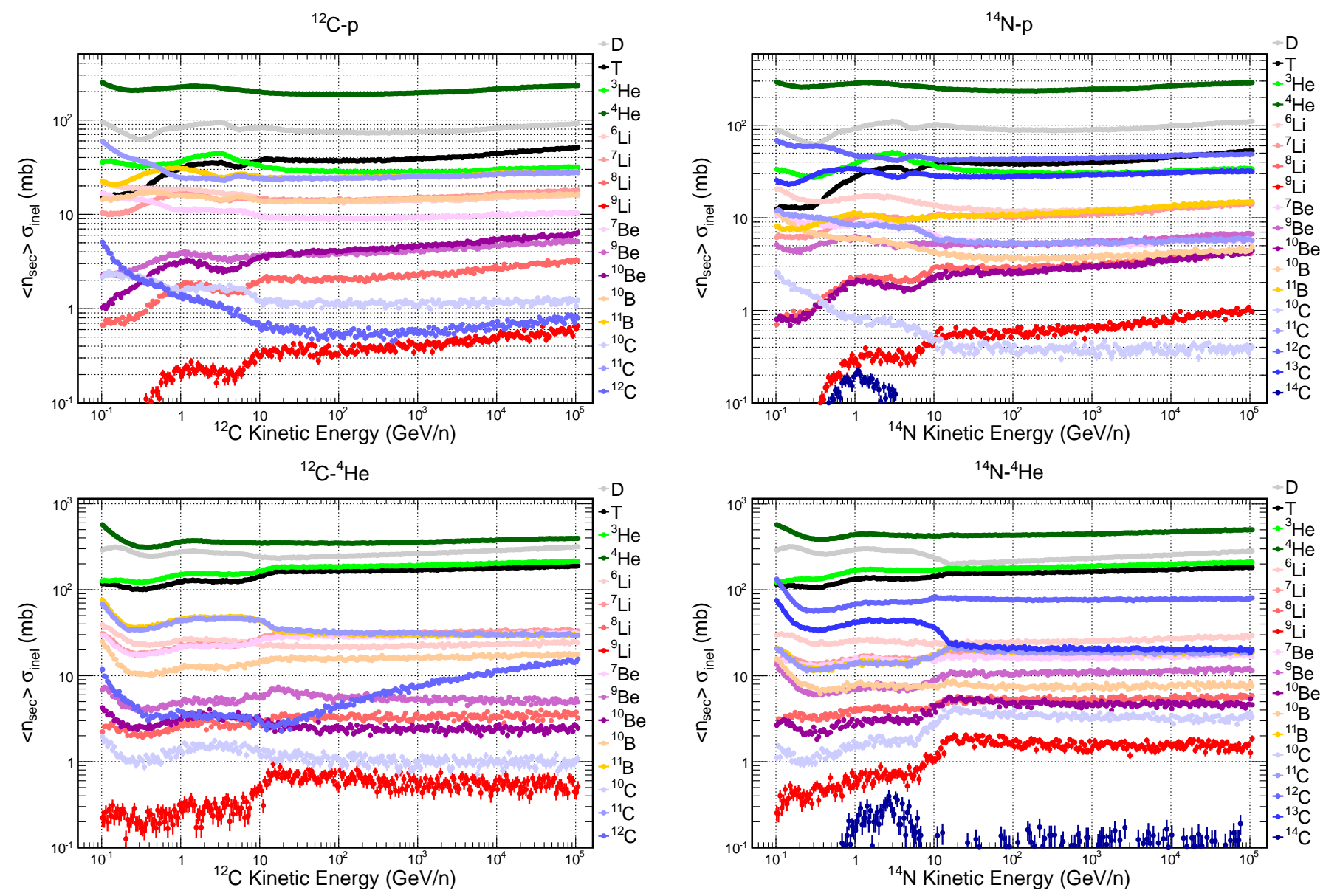

Figure 7: Inclusive cross sections for the production of spallation nuclei in collisions of ${ }^{12} \mathrm{C}$ and ${ }^{14} \mathrm{~N}$ with $\mathrm{p}$ and ${ }^{4} \mathrm{He}$ nuclei. The plots show the cross sections for the production of Deuteron (gray markers), Triton (black markers) and for the isotopes of $\mathrm{He}\left({ }^{3} \mathrm{He}\right.$ and ${ }^{4} \mathrm{He}$, green markers), $\mathrm{Li}\left({ }^{6} \mathrm{Li}\right.$, ${ }^{7} \mathrm{Li},{ }^{8} \mathrm{Li}$ and ${ }^{9} \mathrm{Li}$, red markers), $\mathrm{Be}\left({ }^{7} \mathrm{Be}\right.$ and ${ }^{9} \mathrm{Be}$, magenta markers), $B\left({ }^{10} \mathrm{~B}\right.$ and ${ }^{11} \mathrm{~B}$, orange markers) and $C\left({ }^{10} \mathrm{C},{ }^{11} \mathrm{C},{ }^{12} \mathrm{C},{ }^{13} \mathrm{C}\right.$ and ${ }^{14} \mathrm{C}$, blue markers). Lighter (darker) color shades correspond to lighter (heavier) isotopes.

shown. An agreement at the level of a few percent is obtained in the relevant range of Feynman $X$, both for pion and kaon production. As a further check, in Fig. 3 we show the antiproton production spectrum as a function of the Feynman $X$ for $158 \mathrm{GeV} / \mathrm{c}$ proton on carbon compared with the experimental data [64]. Also in this case a good agreement is found with measured data.

With these results in hand, we can present in full detail all the results of our analysis for all stable particles (including radionuclides whose decay is not taken into account at this stage). First of all we turn our attention on the light products of $p-p, p-H e$ and $\mathrm{He}-\mathrm{He}$ collisions. The species produced in these interactions include a wide range of charged particles and antiparticles, plus gamma rays and neutrinos. Studying these secondary products is extremely important for several applications of CR physics, and also to set contraints on possible exotic contributions (Dark Matter annihilation or decay).

In Figs. 4, 5, A.14, A.15 we show the differential inclusive cross sections for the production of several light secondaries (protons, electrons and positrons, gamma rays, electron neutrinos and antineutrinos, muon neutrinos and antineutrinos, deuterons) in $p-p, p-{ }^{4} \mathrm{He},{ }^{4} \mathrm{He}-{ }^{4} \mathrm{He}$ and $p-{ }^{14} \mathrm{~N}$ interactions as a function of the kinetic energy per nucleon of the projectile and of the kinetic energy of the secondary particle.

As shown in Fig. 5, the inclusive cross section for the production of gamma rays in $p-{ }^{14} N$ collisions exhibits some horizontal lines in the $\mathrm{MeV}$ region, due to nuclear deexcitation processes. This feature is common to all collisions involving heavy nuclei.

The differential inclusive cross sections for the production of electrons, positrons, electron neutrinos and electron antineutrinos shown in Figs. 4, 5, A.14 and A.15 are evaluated taking into account the decay of neutrons and antineutrons. These decays produce low-energy secondaries and provide the main contribution to the inclusive cross sections for secondary energies below a few tens of $\mathrm{MeV}$. Therefore in this region the electron (positron) and the antineutrino (neutrino) cross section will have similar behaviors.

In Figs. 6, A.16 and A.17 we show the total inclusive cross sections for the production of protons, electrons and positrons, gamma rays, electron neutrinos and antineutrinos, muon neutrinos and antineutrinos and Deuteron, as a function of the kinetic energy per nucleon of the projectile in several 
interactions involving different projectiles and targets. As discussed above, the inclusive cross section for the production of electron antineutrinos is similar to that for the production of electrons, and the cross section for the production of positrons is similar to that for the production of electron neutrinos.

In the case of heavy projectile and/or target, the yields of secondary nuclei are not negligible. Some secondary nuclei are unstable: this is for instance the case of Triton, that decays into ${ }^{3} \mathrm{He}+e^{-}+\bar{v}_{e}$ with a half-life of 12.32 years. In our simulation the decay of unstable nuclei produced in spallation processes is not included and must be taken into account when describing CR propagation.

In Fig.s 7 and A.18 we show the most relevant results among all the calculations we performed for the heavy nuclei. We plot the inclusive cross sections related to the the interactions of ${ }^{12} C$, ${ }^{14} \mathrm{~N},{ }^{16} \mathrm{O},{ }^{20} \mathrm{Ne},{ }^{24} \mathrm{Mg}$ and ${ }^{28} \mathrm{Si}$ projectiles with protons and ${ }^{4} \mathrm{He}$ nuclei, yielding deuterons, tritons and all the isotopes of $\mathrm{He}, \mathrm{Li}$, $B e, B$ and $C$.

Concerning ion fragmentation in proton-nucleus collisions, several different verifications of the FLUKA results have been already published elsewhere. For instance, Ref. [43] contains the excitation function of ${ }^{11} C$ for $p+C$ reactions up to $1 \mathrm{GeV}$, while Ref. [46] shows the mass spectrum of fragmentation products from $\mathrm{Pb}+p$ reactions at $1 \mathrm{GeV} / \mathrm{n}$. Furthermore, Ref. [65] covers the high energy side, with mass spectra for $p+A g$ and $p+A u$ reactions at $300 \mathrm{GeV}$ and $800 \mathrm{GeV}$ respectively. Finally, Ref. [66] reports the excitation functions of several fragmentation products from $p+{ }^{59} \mathrm{Co}$ reactions up to $700 \mathrm{MeV}$. Concerning fragmentation in nucleus-nucleus reactions, Ref. [59] contains many charge-changing cross sections for different reactions up to $1 \mathrm{GeV} / \mathrm{n}$, while Ref. [60] does the same for ${ }^{208} \mathrm{~Pb}$ induced reactions at $158 \mathrm{GeV} / \mathrm{n}$.

We remark that this is the first time in which such a complete sample of nuclear cross sections are computed in a consistent way with a single numerical code. Due to the completeness and accuracy of these computations, we point out that the dataset we produced can be used by the community working on $\mathrm{CR}$ physics to constrain the properties of $\mathrm{CR}$ transport in a more solid way, as we will show in the Section 5 .

\section{Evaluation of the energy spectra of secondary cosmic rays}

As a first application, we evaluate the energy spectra of several secondary species produced in the interactions of primary CRs with the ISM. We will consider a simplified CR model in which the differential particle momentum density of any primary species is given by a power law:

$$
n(p)=k_{0}\left(\frac{p}{p_{0}}\right)^{-\alpha}
$$

where $\alpha$ is the spectral index and $k_{0}$ is a normalization constant. In writing the previous equation we introduced a momentum scale $p_{0}=1 \mathrm{GeV}$. Therefore $k_{0}$ will be expressed in the same units as $n(p)$, i.e. in $\mathrm{GeV}^{-1} \mathrm{~m}^{-3}$.

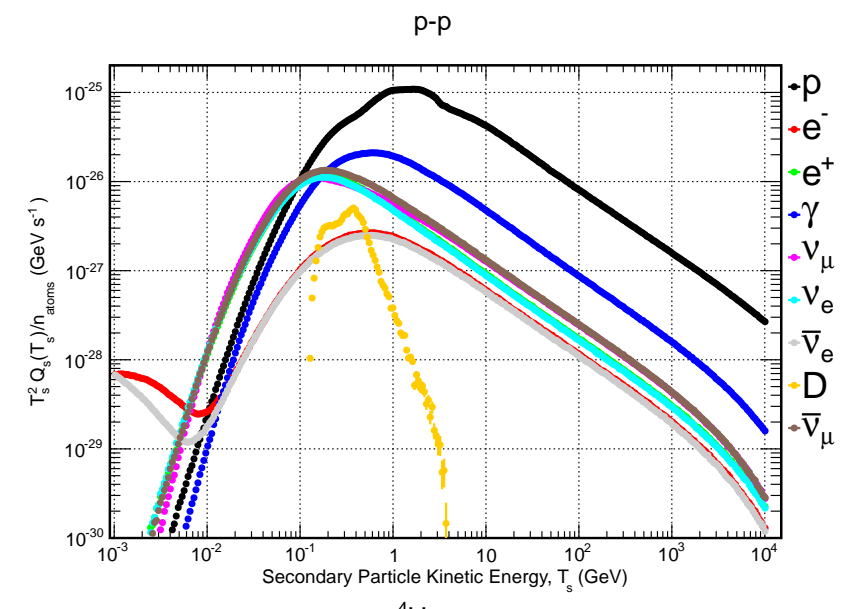

${ }^{4} \mathrm{He}-\mathrm{p}$

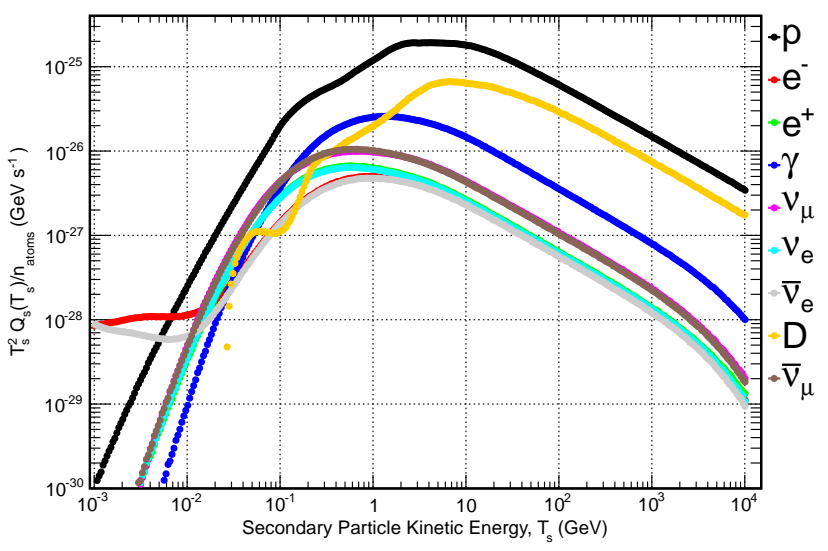

Figure 8: Secondary spectra for proton-proton interaction (top panel) and ${ }^{4} \mathrm{He}$ proton interaction (bottom panel). Protons (black), electrons (red), positrons (green), gamma rays (blue), electron neutrinos (cyan), electron antineutrinos (grey), muon neutrinos (magenta), muon antineutrinos (brown) and Deuterons (orange) The CR proton and Helium intensity is given by the Eq.7 7

The spectral differential intensity in momentum is obtained by multiplying $n(p)$ by the factor $\beta c / 4 \pi$, where $\beta c$ is the particle velocity:

$$
J(p)=\frac{\beta c}{4 \pi} n(p)=k \beta\left(\frac{p}{p_{0}}\right)^{-\alpha} .
$$

where $k=k_{0} c / 4 \pi$ ( $k$ will be expressed in the same units as $J(p)$, i.e. in $\left.\mathrm{GeV}^{-1} \mathrm{~m}^{-2} \mathrm{~s}^{-1} \mathrm{sr}^{-1}\right)$.

The differential intensity in momentum can be converted into a differential intensity in kinetic energy taking into account that:

$$
\begin{gathered}
J(T)=\frac{d p}{d T} J(p) \\
\frac{d p}{d T}=\frac{T+m}{\sqrt{T(T+2 m)}}=\frac{1}{\beta}
\end{gathered}
$$

where $m$ is the rest mass of the particle. The CR spectrum as a function of the kinetic energy is therefore given by:

$$
J(T)=k\left[\frac{T(T+2 m)}{p_{0}^{2}}\right]^{-\frac{\alpha}{2}}
$$


In performing the calculations we assume that the energy spectrum of each primary species is given by:

$$
J(T)=k\left[\frac{T(T+2 m)}{p_{0}^{2}}\right]^{-\frac{\alpha}{2}} e^{-\frac{T}{T_{\max }}} \Theta\left(T_{\max }-T\right) .
$$

The term $\Theta\left(T_{\max }-T\right)$ has been introduced in the formula to take into account that in our simulation we generated primary CRs with energies up to a maximum value $T_{\text {max }}$. We simulated both proton and Helium primaries. For the protons we set $k=2.2 \mathrm{GeV}^{-1} \mathrm{~cm}^{-2} \mathrm{~s}^{-1} \mathrm{sr}^{-1}$ [67] and $\alpha=2.75$, while for the Helium nuclei we set $k=1.0 \mathrm{GeV}^{-1} \mathrm{~cm}^{-2} \mathrm{~s}^{-1} \mathrm{sr}^{-1}$ and $\alpha=2.66$. The cutoff energy value was set at $T_{\max }=10^{5} \mathrm{GeV}$.

The energy spectrum of the secondary particle $s$ is usually expressed in terms of the production function $Q_{s}$, that is defined as:

$$
\frac{Q_{s}\left(T_{s}\right)}{n_{\text {atoms }}}=4 \pi \int J(T) \frac{d \sigma\left(T_{s} \mid T\right)}{d T_{s}} d T
$$

where $n_{\text {atoms }}$ is the number of target atoms per unit volume. The ratio $Q_{s}\left(T_{s}\right) / n_{\text {atoms }}$ is expressed in units of $\mathrm{GeV}^{-1} \mathrm{~s}^{-1}$. Fig. 8 shows the energy spectra of secondaries produced by proton and Helium primaries colliding with proton targets.

\section{Application to the Galactic emission}

In order to evaluate the emission from the Galaxy we use a customized version of the propagation code DRAGON [68, 69] (2D version) $)^{2}$ in which we introduced the present parameterizations in the secondary production routine.

We study the spectra of the following species: protons, Helium, Boron, Carbon, electrons, and positrons; for each one we evaluate the contribution of secondaries originating from nuclear interactions. In our simulation we assume that the ISM is composed of Hydrogen and Helium with relative abundances $1: 0.1$.

Therefore, in the case of leptons, we include the contributions from $\mathrm{p}-\mathrm{p}, \mathrm{p}-{ }^{4} \mathrm{He},{ }^{4} \mathrm{He}-\mathrm{p}$ and ${ }^{4} \mathrm{He}-{ }^{4} \mathrm{He}$ collisions. On the other hand, in the hadronic case, we include all the collisions of $\mathrm{p}, \mathrm{D}, \mathrm{T},{ }^{3} \mathrm{He},{ }^{4} \mathrm{He},{ }^{6} \mathrm{Li},{ }^{7} \mathrm{Li},{ }^{9} \mathrm{Be},{ }^{10} \mathrm{Be}$, ${ }^{10} \mathrm{~B},{ }^{11} \mathrm{~B},{ }^{12} \mathrm{C},{ }^{13} \mathrm{C},{ }^{14} \mathrm{C},{ }^{14} \mathrm{~N},{ }^{15} \mathrm{~N},{ }^{16} \mathrm{O},{ }^{17} \mathrm{O},{ }^{18} \mathrm{O},{ }^{20} \mathrm{Ne},{ }^{24} \mathrm{Mg}$ and ${ }^{28} S i$ on proton and Helium targets. The $\beta^{ \pm}$decays and the electron captures of unstable isotopes are taken into account according to their lifetimes. In the current version of the DRAGON code the daughter nucleus carries out all the energy of the parent nucleus, while the other products (i.e. leptons) are discarded.

The purpose of this section is to discuss the implications of the present parameterizations on some reference diffusion models. The setup we are using is standard: CR transport properties are homogeneous and isotropic; the scalar diffusion coefficient depends on the particle rigidity $R$ and on the distance from the Galactic Plane $z$ according to the following parameterization:

${ }^{2} \mathrm{~A}$ version of DRAGON code is available for download at the link http://www.dragonproject.org/

$$
D=D_{0} \beta^{\eta}\left(\frac{R}{R_{0}}\right)^{\delta} e^{|z| / z_{t}}
$$

where:

- $D_{0}$ is the diffusion coefficient normalization at the reference rigidity $R_{0}=4 \mathrm{GV}$;

- $\eta$ effectively describes the complicated physical effects that may play a major role at low energy (below $1 \mathrm{GeV}$ ), e.g. the dissipation of Alfvén waves due to the resonant interaction with CRs (here $\beta$ represents as usual the particle velocity in units of the speed of light);

- $\delta$ is the spectral index: it is constrained by the data on the light nuclei ratios, in particular by the measurements of the Boron-over-Carbon (B/C) ratio;

- $z_{t}$ is the scale height of the diffusive halo of the Galaxy.

\begin{tabular}{|c|c|c|c|c|}
\hline \hline \multirow{2}{*}{ Nuclei } & \multicolumn{3}{|c|}{ Spectral indices } & \multirow{2}{*}{$\begin{array}{l}\text { Relative } \\
\text { Fraction }\end{array}$} \\
\cline { 2 - 4 } & KOL & KRA & AMS02 & 1 \\
\hline${ }^{1} H$ & $2.05 / 2.48$ & $2.05 / 2.33$ & $2.05 / 2.43$ & $10.13 \times 10^{-2}$ \\
\hline${ }^{4} \mathrm{He}$ & $2.18 / 2.40$ & $2.18 / 2.24$ & $2.18 / 2.32$ & $6.13 \times 10^{-3}$ \\
\hline${ }^{12} \mathrm{C}$ & $2.20 / 2.60$ & $2.20 / 2.40$ & $2.20 / 2.50$ & $3.11 \times 10$ \\
\hline${ }^{14} \mathrm{~N}$ & $2.20 / 2.60$ & $2.20 / 2.40$ & $2.20 / 2.50$ & $2.16 \times 10^{-4}$ \\
\hline${ }^{16} \mathrm{O}$ & $2.20 / 2.60$ & $2.20 / 2.40$ & $2.20 / 2.50$ & $3.61 \times 10^{-3}$ \\
\hline${ }^{20} \mathrm{Ne}$ & $2.20 / 2.60$ & $2.20 / 2.40$ & $2.20 / 2.30$ & $2.94 \times 10^{-4}$ \\
\hline${ }^{24} \mathrm{Mg}$ & $2.20 / 2.60$ & $2.20 / 2.40$ & $2.20 / 2.50$ & $6.21 \times 10^{-4}$ \\
\hline${ }^{28} \mathrm{~S} i$ & $2.20 / 2.60$ & $2.20 / 2.40$ & $2.20 / 2.50$ & $6.85 \times 10^{-4}$ \\
\hline$e^{-}$ & $1.60 / 2.50$ & $1.60 / 2.50$ & $1.60 / 2.50$ & \\
\hline Extra $e^{+}$ & 1.78 & 1.78 & 1.78 & \\
\hline \hline
\end{tabular}

Table 1: Main parameters of the injection spectra of the primary species.

Our starting points consist of two diffusion models considered as a reference in several previous works (see e.g. [76]) labelled as KRA and KOL: they are mainly tuned on PAMELA B/C data. We also present a new model tuned on the recent preliminary AMS02 data [77]. In the following we summarize the main features of these models:

- KRA features $\delta=0.5$ (compatible with a Kraichnan turbulence in the framework of Quasi-Linear Theory), a moderate level of reacceleration (The Alfvén velocity is $\simeq 15 \mathrm{~km} / \mathrm{s}$ ), and an altered diffusion coefficient at low energy parametrized by $\eta \simeq-0.4$.

- KOL is based on $\delta=0.33$ (compatible with a Kolmogorov turbulence in the framework of Quasi-Linear Theory), a high level of reacceleration (The Alfvén velocity is $\simeq$ $30 \mathrm{~km} / \mathrm{s}$ ).

- The model tuned on recent preliminary AMS02 data has $\delta=0.42$ and the same Alfvén velocity as the KRA model. This model is based on the one presented in [78] 

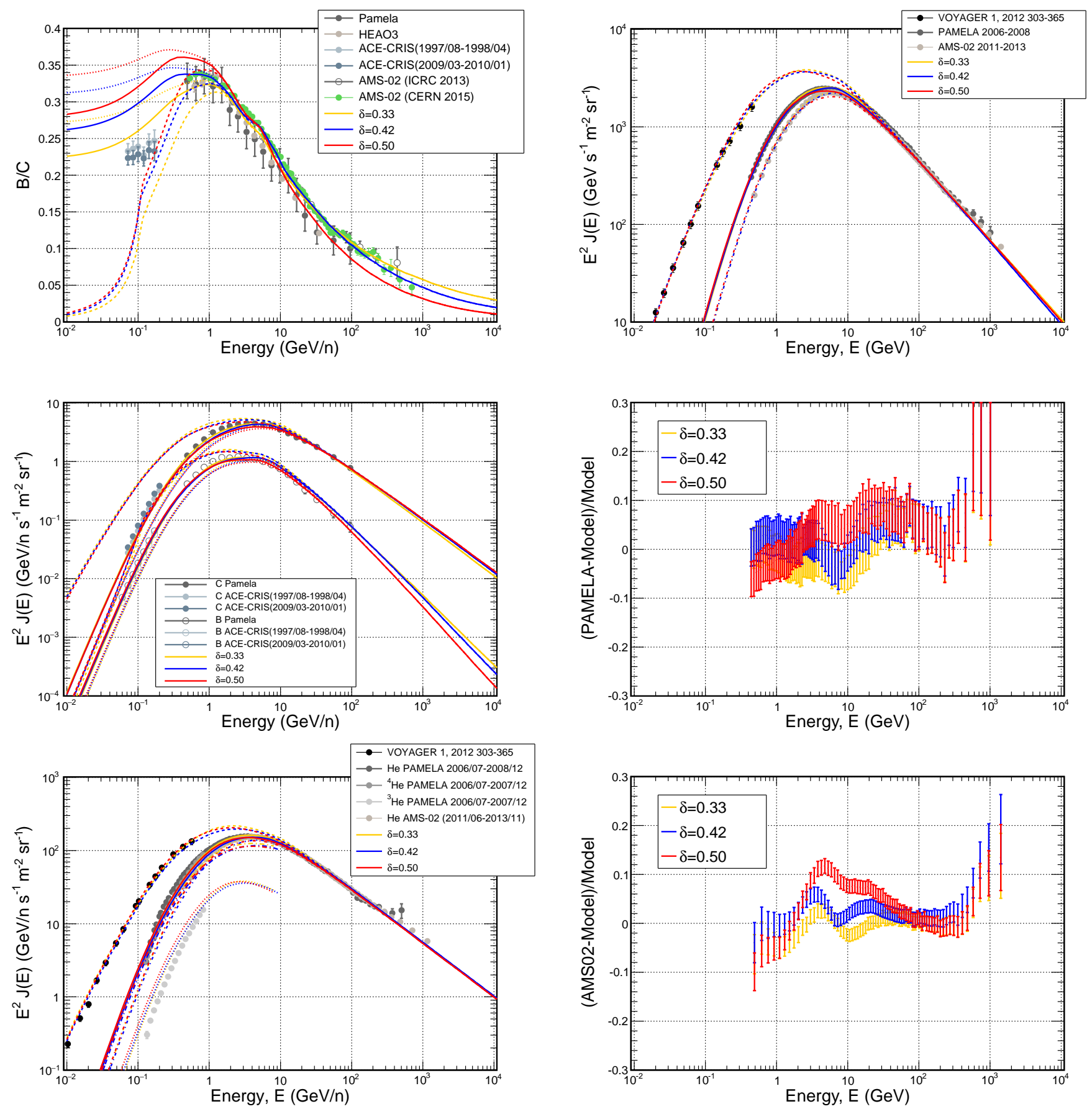

Figure 9: DRAGON results. The models are: $\delta=0.33$ (KOL), $\delta=0.42$ and $\delta=0.50$ (KRA). Left, from top to bottom: Boron to Carbon ratio, Carbon and Boron and Helium. Right, from top to bottom, proton comparison with PAMELA and AMS02 data. Dashed line: unmodulated intensity: solid (dotted) line: modulated intensity by means of the force field approximation with $\phi=0.42$ (0.62) GV. Fractional residual betweet PAMELA (middle) and AMS02 (bottom). VOYAGER1 [82]; PAMELA [13] 19]; ACE-CRIS [83]; AMS01 [85]; HEAO3-C2 [84] (some data points are quoted from the Database of Charged Cosmic Rays [86])

In all cases the injected spectra up to Silicon nuclei are described by a broken power law model; the main parameters describing the injection spectra are summarized in Table 1 . The source term distrubution is taken from [53]. The gas density distribution is taken from the public Galprop version [87, 88, 89].
We also consider a primary electron+positron extra component with harder injection spectrum in order to explain the anomalous rise of the positron fraction above $10 \mathrm{GeV}$ reported by PAMELA, Fermi-LAT and AMS02. This behaviour cannot be reproduced in the standard scenario in which positrons only originate from spallation of protons, 

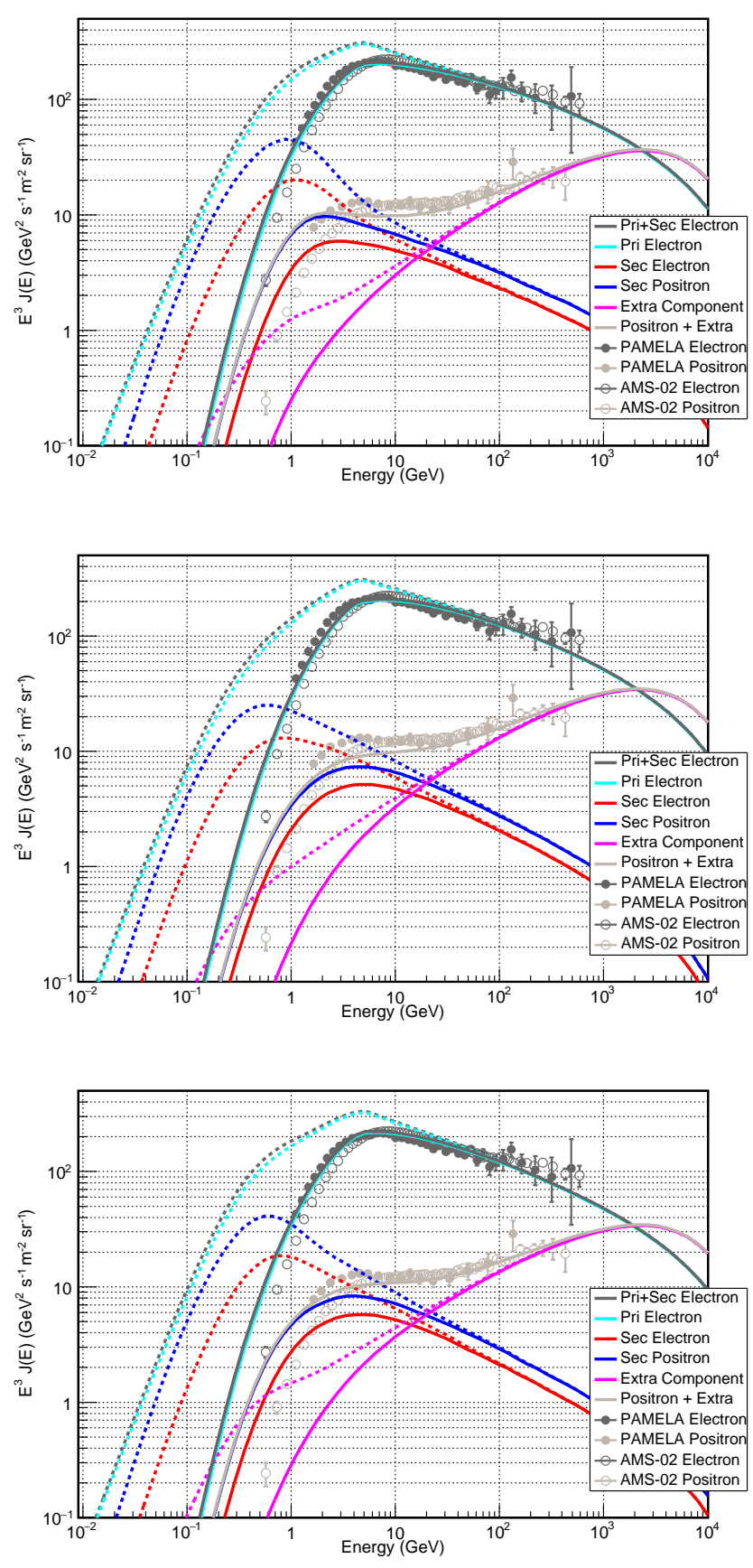

Figure 10: DRAGON results for electrons and positrons. The models are (from top to bottom): $\delta=0.33$ (KOL), $\delta=0.42$ and $\delta=0.50$ (KRA). Dashed line: unmodulated intensity: solid line: modulated intensity by means of the force field approximation with $\phi=0.42 \mathrm{GV}$ (some data point are quoted from the Database of Charged Cosmic Rays [86])

helium and heavier nuclei on interstellar gas.

In order to compare our computations with the data, we have to consider that, in the final stage of the propagation, charged particles enter the sphere of influence of the Sun. Here they diffuse in the Heliospheric magnetic field, and suffer adiabatic energy losses and convection due to the presence of the solar

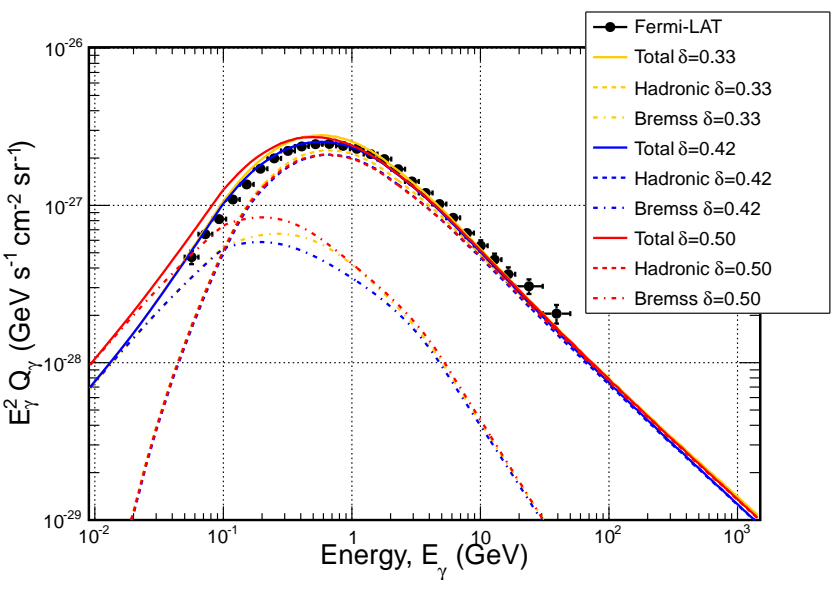

Figure 11: Differential gamma-ray emissivity as a function of the gamma-ray energy. We calculated the contribution associated with the hadronic collisions and bremsstrahlung by folding the proton, the Helium and electron LIS with $\gamma$-ray production cross sections. Orange: KOL model; Red: KRA model; Blue: AMS02 model (see text). The data point are quoted from [73].

wind: this process is called solar modulation and is relevant for low energy $(<10 \mathrm{GeV})$ particles. In this work we adopt the so-called "force field approximation" [70], a simplified description in which a single free parameter is involved: the modulation potential $\phi$.

Given all these ingredients, our method is the following: we compare the current data to the computations performed with our modified version of DRAGON with the new cross section parametrizations and we retune the model accordingly. Our results are summarized in Fig.s 9 and 10 Very remarkably, we find that it is possible to reproduce correctly all the relevant observables (namely, proton, Helium, light nuclei spectra, and $\mathrm{B} / \mathrm{C}$ ratio) with the reference models considered, after a rescaling of the diffusion coefficient normalization $\left(D_{0}\right)$ and a minor fine-tuning of the other parameters.

- For the KOL model we use $D_{0}=4.25 \times 10^{28} \mathrm{~cm}^{2} \mathrm{~s}^{-1}$, $\eta=0$ and $v_{\mathrm{A}}=33 \mathrm{~km} \mathrm{~s}^{-1}$ (Alfvén velocity): while

- For the KRA model we use $D_{0}=2.8 \times 10^{28} \mathrm{~cm}^{2} \mathrm{~s}^{-1}$, $\eta=-0.4$ and $v_{\mathrm{A}}=17.5 \mathrm{~km} \mathrm{~s}^{-1}$.

- For the AMS02 model we use $D_{0}=3.35 \times 10^{28} \mathrm{~cm}^{2} \mathrm{~s}^{-1}$, $\eta=-0.4$ and $v_{\mathrm{A}}=17.5 \mathrm{~km} \mathrm{~s}^{-1}$.

For the three models we use the solar modulation parameter $\phi=0.42$ and $0.62 \mathrm{GV}$ for all the species to reproduce the PAMELA and AMS02 data respectively. The comparison of these results with PAMELA 3 and AMSO2 proton data is within $\pm 10 \%$ or better, as shown in Fig. 9 Clearly, the model with $\delta=0.42$ is designed to have the best compatibility with AMSO2 preliminary $\mathrm{B} / \mathrm{C}$ data.

We remind that in the low energy region it is useful in many cases to go beyond the very simplified picture of the force field

\footnotetext{
${ }^{3}$ We lowered the proton spectrum by $3.2 \%$ according to the prescription in Ref. [18].
} 
approximation, since the modulation process depends on the charge of the particle (including its sign). A more accurate treatment consists in using a dedicated numerical transport tool to describe the propagation process in the Solar System, e.g. HelioProp [71], but that is far beyond the scope of this paper.

It is worth to point out that in these three models we do not consider the presence of the break around $300 \mathrm{GeV}$ in the proton and nuclei spectra, since it is beyond the aim of this paper. However, with this complete sample of inclusive cross sections we do not need to introduce the concept of nuclear enhancement factor to describe secondary production in interaction among heavier nuclei, extrapolating the results of $p-p$ interaction [35]. In particular, this factor depends on the shape of the spectrum (see for instance [72]) and it does not work in case of abrupt change in the CR spectra (as in case of breaks).

Here we want to stress that with this new evaluation we have a much better insight on the nuclear processes, since the crosssections can be computed accurately in a wide energy range. For this reason, our approach opens the possibility of more detailed studies on the CR spectra, especially in the low-energy range (below $10 \mathrm{GeV}$ ).

For example, given the more accurate determination of the gamma-ray emissivity, we can turn our attention to the Local Interstellar Spectra (LIS) of protons and Helium nuclei, not affected by solar modulation. This quantity can be inferred from the Fermi-LAT gamma-ray data [73] and its determination is useful in order to constrain the propagation models described above, since each of them implies a different shape and normalization for the LIS. Fig. 11 shows the gamma-ray emissivity calculated by means of the proton, Helium and electron LIS. We calculate the contribution associated with the hadronic collisions and bremsstrahlung by folding the proton, Helium and electron LIS with the corresponding $\gamma$-ray production cross sections (according to Eq. 8) on the ISM with relative abundance of $H: H e: C: N: O: N e: M g: S i=1$ : $0.096: 4.65 \times 10^{-4}: 8.3 \times 10^{-5}: 8.3 \times 10^{-4}: 1.3 \times 10^{-4}: 3.9 \times$ $10^{-5}: 3.69 \times 10^{-5}$. An inspection of Fig. 11 shows that at low energies the contribution from electron bremsstrahlung is not negligible. This contribution mainly depends on the electron spectrum that should also be constrained taking synchrotron radiation into account (see for instance [74, 75]).

In order to test our model even further, we evaluate the Galactic diffuse gamma-ray and neutrino emission adopting the numerical package GammaSky as post-processor of the DRAGON output. GammaSky is a dedicated code recently used e.g. in [76, 79, 80] and other works to compute gammaray, synchrotron and neutrino maps and spectra. This package includes, among the different options, the gas model taken from the public version of Galprop (see e.g. [90, 91] and references therein).

We implement in GammaSky the present parameterizations for the gamma-ray and neutrino secondary production in the hadronic interactions (taking into account the $p-p, p-{ }^{4} \mathrm{He}$, ${ }^{4} \mathrm{He}-\mathrm{p}$ and ${ }^{4} \mathrm{He}-{ }^{4} \mathrm{He}$ collisions with ISM and assuming an ISM $H: H e=1: 0.1$ ).

Fig. 12 shows the sky maps of the integral gamma- ray intensity above $10 \mathrm{MeV}$ for the various components. The hadronic contribution is evaluated using the present parameterizations, while the bremsstrahlung and inverse Compton contributions are evaluated using the built-in models in GammaSky. The last panel of Fig. 12 shows the average gamma-ray intensities as a function of the energy for the different components. The calculation is performed considering either the whole sky or only the region outside the galactic plane $\left(|b|>10^{\circ}\right)$. In both cases the contribution from the hadronic component becomes dominant in the energy range above $100 \mathrm{MeV}$.

Fig. 13 shows the maps of the integral intensities above $10 \mathrm{MeV}$ of the various species of neutrinos and antineutrinos. The bottom panels of Fig. 13 show the average differential intensity of electron and muon neutrinos and antineutrinos, evaluated considering either the whole sky or only the region outside the galactic plane $\left(|b|>10^{\circ}\right)$.

\section{Conclusions}

We have used the FLUKA simulation code to evaluate the inclusive cross sections for the production of stable secondary particles and as well as nuclei from spallation in the interactions of several CR projectiles with different target nuclei.

The results are provided in tables, that can be used for the computation of the production spectra of secondary CRs starting from any given spectra of the primaries. The propagation and the interactions of these particles in astronomical environments can then be studied using dedicated tools, as DRAGON, GammaSky and Galprop.

As an example of application we implemented the values of the cross sections in a custom version of the DRAGON CR propagation code, showing that it is possible to reproduce with accuracy the measured CR spectra and light nuclei ratios as well as the gamma-ray emissivity. In the present work we have propagated only $\mathrm{CR}$ projectiles up to $Z=14$ negleting heavier nuclei that could affect the present results at the level of a few percent. The production and the propagation of nuclei up to iron will be investigated in a future work.

Finally, we evaluated the gamma-ray and neutrino skymaps and energy distributions using a custom version of the GammaSky code.

[1] A. Putze, L. Derome and D. Maurin, Astron. Astrophys. 516 (2010) A66 arXiv:1001.0551 [astro-ph.HE]].

[2] G. Di Bernardo, C. Evoli, D. Gaggero, D. Grasso and L. Maccione, Astropart. Phys. 34, 274 (2010) |arXiv:0909.4548 [astro-ph.HE]].

[3] R. Trotta, G. Johannesson, I. V. Moskalenko, T. A. Porter, R. R. de Austri and A. W. Strong, Astrophys. J. 729, 106 (2011) |arXiv:1011.0037] [astroph.HE]].

[4] W.B. Atwood at al. (The Fermi LAT Collaboration), Astrophysical Journal 697 (2009) 1071

[5] F. Aharonian et al. (The H.E.S.S. Collaboration), Astroparticle Physics 22 (2004) 109

[6] J. Aleksić et al. (The MAGIC Collaboration), Astroparticle Physics 35 (2012) 435

[7] V.A. Acciari et al. (VERITAS Collaboration), Astrophysical Journal 679 (2008) 1427

[8] M. Actis et al. (The CTA Consortium), Exp. Astron. 32 (2011) 193

[9] M. Ackermann et al. [Fermi-LAT Collaboration], Astrophys. J. 750 (2012) 3 

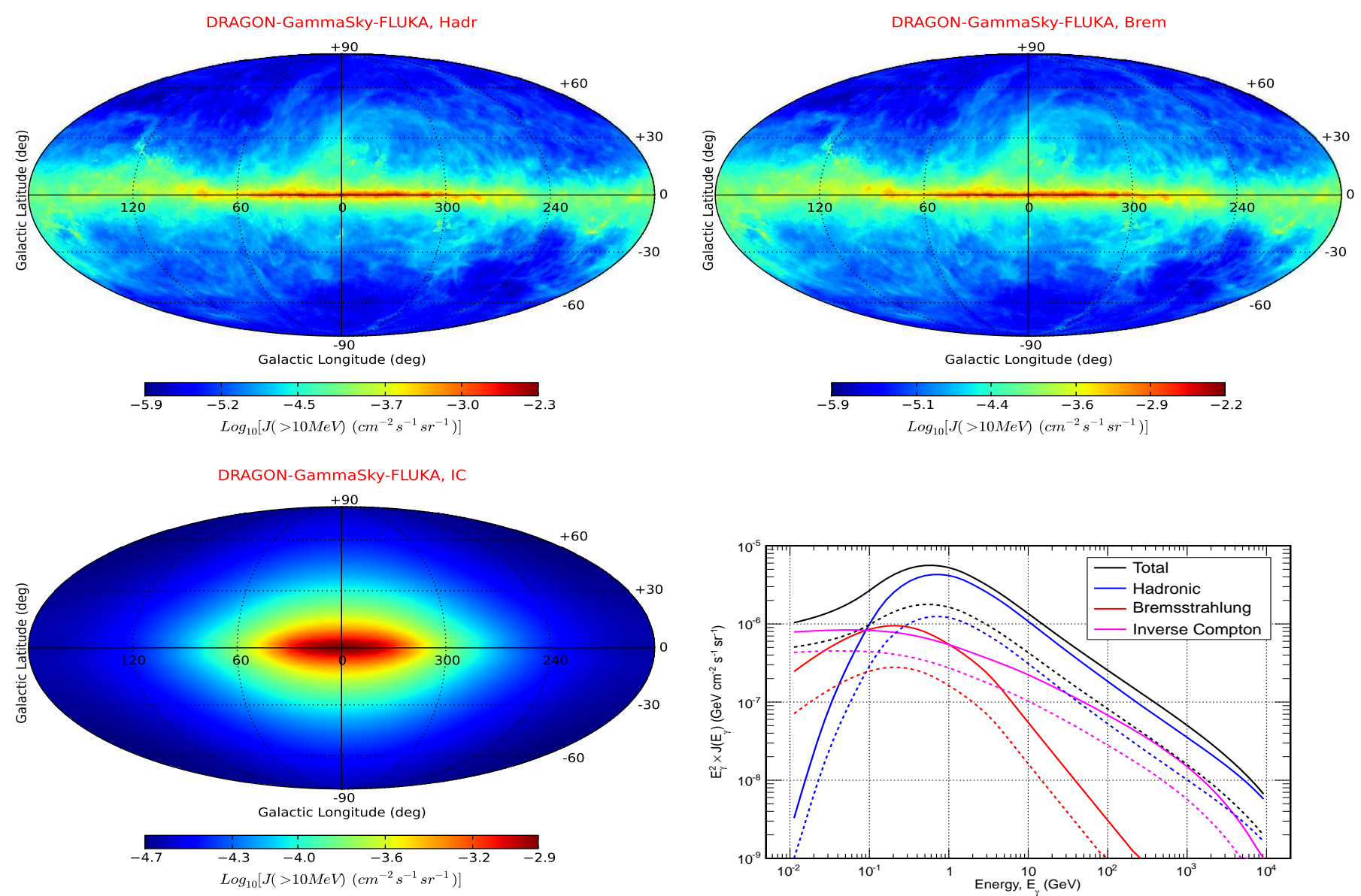

Figure 12: Gamma-ray results obtained with GammaSky. The top left, top right and bottom left plots show the sky maps of the integral gamma-ray intensities above $10 \mathrm{MeV}$ for the hadronic, bremsstrahlung and inverse Compton (IC) emission. The maps have been built using the HEALPix [81] pixelization in the Galactic reference frame (Mollweide projection). The bottom right plot shows the differential average intensities multiplied by $E_{\gamma}^{2}$ as a function of the gamma-ray energy. The solid lines have been obtained summing the intensities over the whole sky, the dashed lines refer to the region $|b|>10^{\circ}$.

[10] C.Evoli et al., Physical Review Letters 108, 21 (2012) 211102

[11] D.Gaggero et al., submitted to Physical Review Letters |arXiv:1411.7623 [astro-ph]]

[12] M. Ackermann et al. [Fermi-LAT Collaboration], Phys. Rev. Lett. 112, 151103 (2014) arXiv:1403.5372 [astro-ph.HE]].

[13] O. Adriani et al. [PAMELA Collaboration], Science 332 (2011) 69 [arXiv:1103.4055 [astro-ph.HE]].

[14] M. Aguilar [AMS Collaboration], Phys. Rev. Lett. 114, no. 17, 171103 (2015).

[15] M. Aguilar [AMS Collaboration], Phys. Rev. Lett. 115, 211101 (2015).

[16] O. Adriani et al. [PAMELA Collaboration], Nature 458 (2009) 607 |arXiv:0810.4995 [astro-ph]].

[17] O. Adriani et al. [PAMELA Collaboration], Phys. Rev. Lett. 111 (2013) 8, 081102 [arXiv:1308.0133 [astro-ph.HE]].

[18] O. Adriani, G. C. Barbarino, G. A. Bazilevskaya, R. Bellotti, M. Boezio, E. A. Bogomolov, M. Bongi and V. Bonvicini et al., Astrophys. J. 765, 91 (2013) [arXiv:1301.4108 [astro-ph.HE]]

[19] O. Adriani et al. [PAMELA Collaboration], Astrophys. J. 770 (2013) 2 arXiv:1304.5420 [astro-ph.HE]].

[20] O. Adriani et al. [PAMELA Collaboration], Phys. Rev. Lett. 106 (2011) 201101 [arXiv:1103.2880 [astro-ph.HE]].

[21] O. Adriani et al. [PAMELA Collaboration], Phys. Rev. Lett. 105 (2010) 121101 |arXiv:1007.0821 [astro-ph.HE]].

[22] M. Aguilar et al. [AMS Collaboration], Phys. Rev. Lett. 110 (2013) 14, 141102

[23] L. Accardo et al. [AMS Collaboration], Phys. Rev. Lett. 113, 121101
(2014).

[24] M. Aguilar et al. [AMS Collaboration], Phys. Rev. Lett. 113 (2014) 121102.

[25] http://www.ams02.org/2013/07/new-results-from-ams-presented-at-icrc2013/

[26] http://www.ams02.org/2015/04/ams-days-at-cern-and-latest-resultsfrom-the-ams-experiment-on-the-international-space-station/ https://indico.cern.ch/event/381134/otherview? view $=$ standard\#20150415

[27] F.W. Stecker, Astrophysics and Space Science 6 (1970) 377

[28] C.D. Dermer, Astrophysical Journal 307 (1986) 47

[29] I.V. Moskalenko and A.W. Strong, Astrophysical Journal 493 (1998) 694

[30] K.A. Olive et al. (Particle Data Group), Chin. Phys. C, 38 (2014) 090001

[31] C.-Y. Huang et al, Astroparticle Physics 27 (2007) 429

[32] M. Mori, Astrophysical Journal 225 (1997) 225

[33] T. Kamae et al., Astrophysical Journal 708 (2006) 647

[34] S.R. Kelner et al., Phys. Rev. D 74 (2006) 034018

[35] M. Mori, Astroparticle Physics 31 (2009) 341

[36] M. Kachelrieß and S. Ostapchenko, Phys. Rev. D 84 (2012) 043004

[37] T. Sjostrand, Computer Physics Commun. 82 (1994) 74

[38] S. Roesler, R. Engel and J. Ranft, The Monte Carlo event generator DPMJET-III Proc. Monte Carlo 2000 Conference, Lisbon, October 2326 2000, A. Kling, F. Bar ao, M. Nakagawa, L. T'avora, P. Vaz eds., Springer-Verlag Berlin, p. 1033-1038, 2001

[39] R. S. Fletcher, T.K. Gaisser, P. Lipari, and T. Stanev, Phys. Rev. D 50 (1994) 5710 

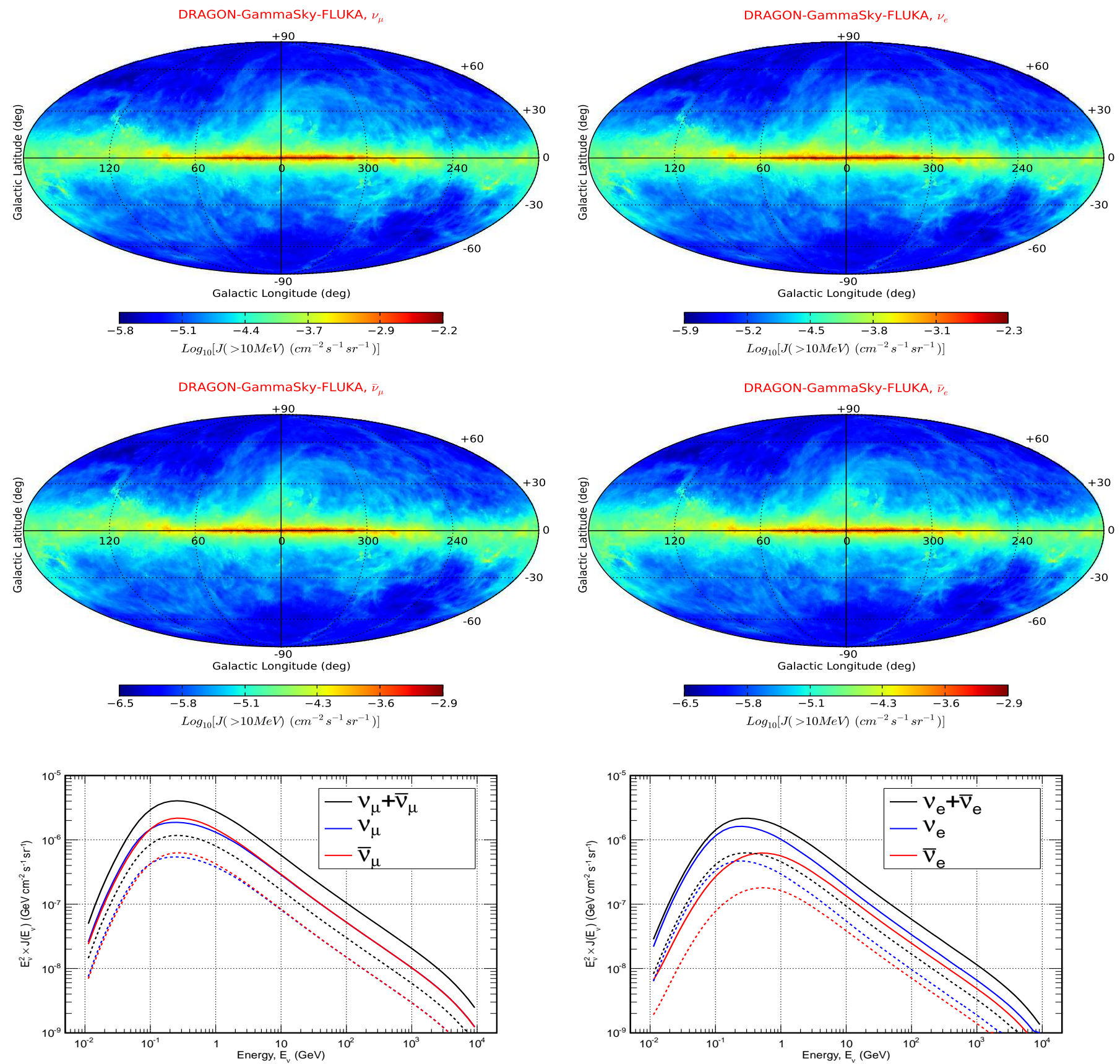

Figure 13: Neutrino results obtained with GammaSky. The plots in the left column refer to muon neutrinos and antineutrinos; the plots in the right column refer to electron neutrinos and antineutrinos. The four top panels show the sky maps of the integral intensities above $10 \mathrm{MeV}$. The maps are in Galactic coordinates and have been built using the HEALPix pixelization scheme (Mollweide projection). The bottom plots show the differential average intensities multiplied by $E_{v}^{2}$ as a function of the neutrino energy. The solid lines have been obtained summing the intensities over the whole sky, the dashed lines refer to the region $|b|>10^{\circ}$.

[40] S. Ostapchenko, Phys. Rev. D 83 (2011) 014018

[41] A. Ferrari, P.R. Sala, A. Fassò, and J. Ranft, FLUKA: a multi-particle transport code CERN-2005-10 (2005), INFN/TC_05/11, SLAC-R-773

[42] http://www.fluka.org

[43] T. Bohlen, F. Cerutti, M.P.W. Chin, A. Fassò, A. Ferrari, P.G. Ortega, A. Mairani, P.R. Sala, G. Smirnov, and V. Vlachoudis, The FLUKA Code: Developments and Challenges for High Energy and Medical Applications, Nuclear Data Sheets 120 (2014) 211-214

[44] F. Ballarini et al., Nuclear models in FLUKA: a review, Proceedings 10th International Conference on Nuclear Reaction Mechanisms, Varenna,
Italy, June 9-13, 2003, edited by E. Gadioli, Ric. Scient. ed Educ. Perm. S122, Univ. degli Studi di Milano (2003) 579-591, www.mi.infn.it/ radioli/Varenna2006/Proceedings/Ferrari_A.pdf

[45] G. Battistoni et al., The FLUKA code: Description and benchmarking, Proceedings of the Hadronic Shower Simulation Workshop 2006, Fermilab 6-8 September 2006, M. Albrow, R. Raja eds., AIP Conference Proceeding 896 (2007) 31-49

[46] G. Battistoni, T. Boehlen, F. Cerutti, P. Wai Chin, L. S. Esposito, A. Fassò, A. Ferrari, A. Lechner, A. Empl, A. Mairani, A. Mereghetti, P. G. Ortega, J. Ranft, S. Roesler, P. R. Sala, V. Vlachoudis, and G. Smirnov, Overview 
of the FLUKA code, Annals of Nuclear Energy 82 (2015) 10-18

[47] A. Fassò, A. Ferrari, J. Ranft, P.R. Sala, FLUKA: status and prospective for hadronic applications, in: A. Kling, F. Barão, M. Nakagawa, L. Távora, P. Vaz (Eds.) Proceedings of the Monte Carlo 2000 Conference, Lisbon, October 2326 2000, Springer-Verlag, Berlin, 2001, p. 955960

[48] G. Battistoni et al. Recent developments in the FLUKA nuclear reaction models Proc. 11th Int. Conf. on Nucl. React. Mech. (Varenna, Italy, 1216 June 2006) 483

[49] A. Ferrari and P.R. Sala The Physics of High Energy Reactions, Proc. Workshop on Nuclear Reaction Data and Nuclear Reactors Physics, Design and Safety, International Centre for Theoretical Physics, Miramare-Trieste, Italy, 15 April-17 May 1996, Ed. A. Gandini and G. Reffo, World Scientific, p. 424 (1998) https://cds.cern.ch/ record/682497

[50] G. Battistoni, A. Ferrari, T. Montaruli and P.R. Sala, Astroparticle Physics 17 (2002) 477

[51] G. Battistoni, A. Ferrari, T. Montaruli and P.R. Sala, Astroparticle Physics 19 (2003) 269

[52] G. Battistoni, A. Ferrari, T. Montaruli and P.R. Sala, Astroparticle Physics 23 (2005) 526

[53] K. M. Ferriere, Rev. Mod. Phys. 73, 1031 (2001) astro-ph/0106359].

[54] R. Ramaty, B. Kozlovsky and R.E. Lingenfelter, Astrophysical Journal Supplement Series 40 (1979) 487

[55] V. Andersen et al., Advances in Space Research 34 (2004) 1302

[56] H. Sorge, H. Stoecker and W. Greiner, Ann. Phys. 192 (1989) 266

[57] H. Sorge, H. Stoecker and W. Greiner, Nucl. Phys. A498 (1989) 567

[58] H. Sorge, Phys. Rev. C52 (1995) 3291

[59] L. Sihver et al., Benchmarking of calculated projectile fragmentation cross-sections using the 3-D, MC codes PHITS, FLUKA, HETC-HEDS, MCNPX HI, and NUCFRG2, Acta Astronautica 63 (2008) 865

[60] H.H. Braun et al., Hadronic and electromagnetic fragmentation of ultrarelativistic heavy ions at LHC, Phys. Rev. Special Topics Accelerators and Beams 17, 021006 (2014)

[61] A. Fedynitch and R. Engel, Revision of the high energy hadronic interaction models PHOJET/DPMJETIII, Proceedings of the 14th International Conference on Nuclear Reaction Mechanisms, edited by F. Cerutti, M. Chadwick, A. Ferrari, T. Kawano and P. Schoofs, CERNProceedings-2015-001 (CERN, Geneva, 2015), 291-299, https : //cds . cern.ch/record/2114737

[62] C. Alt et al. [NA49 Collaboration], Eur. Phys. J. C 49 (2007) 897 doi:10.1140/epjc/s10052-006-0165-7 [hep-ex/0606028].

[63] T. Anticic et al. [NA49 Collaboration], Eur. Phys. J. C 68 (2010) doi:10.1140/epjc/s10052-010-1328-0 [arXiv:1004.1889 [hep-ex]].

[64] B. Baatar et al. [NA49 Collaboration], Eur. Phys. J. C 73 (2013) no.4, 2364 doi:10.1140/epjc/s10052-013-2364-3 [arXiv:1207.6520 [hep-ex]].

[65] F. Ballarini et al., AIP Conf. Proc. 769 (2005) 1197. doi:10.1063/1.1945222

[66] A. Ferrari and P.R. Sala Intermediate and High Energy Models in FLUKA: Improvements, Benchmarks and Applications, Proceedings of the International Conference on Nuclear Data for Science and Technology (NDST-97), International Centre for Theoretical Physics, MiramareTrieste, Italy, May 19-24 1997, G. Reffo, A. Ventura and C. Grandi eds., Italian Physical Society publ., ISBN 88-7794-114-6, Bologna, Part I, (1997) 247-253, available at http://www.fluka.org/content/ publications/1997_trieste.pdf

[67] C. D. Dermer, Phys. Rev. Lett. 109 (2012), 091101

[68] C. Evoli, D. Gaggero, D. Grasso and L. Maccione, JCAP 0810 (2008) 018 |arXiv:0807.4730 [astro-ph]].

[69] D. Gaggero, L. Maccione, G. Di Bernardo, C. Evoli and D. Grasso, Phys. Rev. Lett. 111 (2013) 2, 021102 |arXiv:1304.6718 [astro-ph.HE]].

[70] L.J. Gleeson and W.I. Axford, ApJ 149, L115 (1967) and L.J. Gleeson and W.I. Axford, ApJ 154, 1011 (1968).

[71] L. Maccione, Phys. Rev. Lett. 110 (2013) 8, 081101 |arXiv:1211.6905 [astro-ph.HE]].

See also: D. Gaggero, D. Grasso, L. Maccione, G. Di Bernardo and C. Evoli, Phys. Rev. D 89 (2014) 083007 arXiv:1311.5575 [astroph.HE]].

[72] M. Kachelriess, I. V. Moskalenko and S. S. Ostapchenko, Astrophys. J. 789 (2014) 136 [arXiv:1406.0035 [astro-ph.HE]].

[73] J.M. Casandjian, Astrophysical Journal $806 \quad$ (2015) 240 [arXiv:1506.00047[astro-ph.HE]]
[74] G. Di Bernardo, C. Evoli, D. Gaggero, D. Grasso and L. Maccione, JCAP 1303 (2013) 036 |arXiv:1210.4546 [astro-ph.HE]]

[75] E. Orlando and A. Strong, MNRAS 436 (2013) 2127 |arXiv:1309.2947 [astro-ph.GA]]

[76] C. Evoli, D. Gaggero, D. Grasso and L. Maccione, Phys. Rev. Lett. 108, 211102 (2012) [arXiv:1203.0570 [astro-ph.HE]]

[77] http://www.ams02.org/2015/04/

[78] C. Evoli, D. Gaggero, and D. Grasso |arXiv:1504.05175 [astro-ph.HE]]

[79] M. Tavakoli, I. Cholis, C. Evoli and P. Ullio, JCAP 01 (2014) id. 017 arXiv:1110.5922 [astro-ph.HE]

[80] M. Cirelli, D. Gaggero, G. Giesen, M. Taoso and A. Urbano, JCAP 12 (2014) id. 045 arXiv:1407.2173 [hep-ph]

[81] K.M. Górski, E. Hivon, A.J. Banday, B.D. Wandelt, F.K. Hansen, M. Reinecke, and M. Bartelmann, ApJ 622 (2005) 759-771; http:// healpix.jpl.nasa.gov

[82] E.C. Stone et al., Science 341 (2013) 150

[83] K.A. Lave et al., ApJ 770 (2013) 117

[84] J.J. Engelmann et al., A\&A 233 (1990) 96

[85] M. Aguilar et al. (AMS Collaboration), Phys. Reports 366, 331 (2002)

[86] D. Maurin, F. Melot and R. Taillet, A\&A 569 (2014) A32 arXiv:1302.5525]

[87] http://galprop.stanford.edu/

[88] A.W.Strong and I.V. Moskalenko, ApJ, 509, Issue 1, pp. 212-228 (1998)

[89] I.V. Moskalenko et al., ApJ, 565, Issue 1, pp. 280-296 (2002)

[90] A.W. Strong, I.V. Moskalenko, and V.S. Ptuskin., Ann.Rev.Nucl.Part.Sci., 57:285327 (2007)

[91] M. Ackermann et al. (Fermi-LAT collaboration), ApJ 750 (2012)

\section{Appendix A. Additional plots}

In this section we present additional results of inclusive secondary cross sections. 

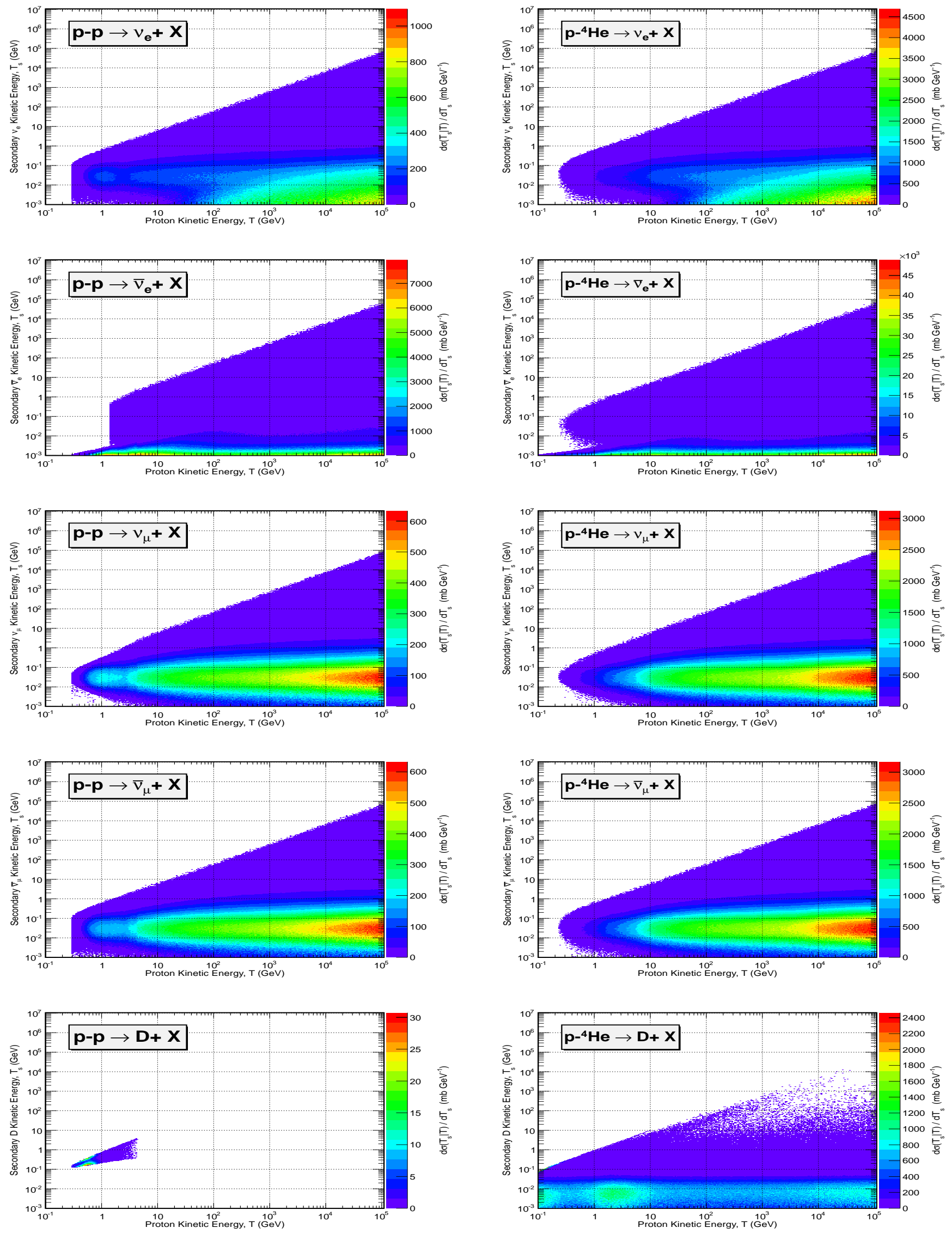

Figure A.14: Differential inclusive secondary cross sections for the production of $v, \bar{v}$ and deuteron in $p-p$ (left) and $p-{ }^{4} \mathrm{He}$ (right) interactions. The values of the cross sections (color scales) are in $\mathrm{mb} \mathrm{GeV}^{-1}$. 

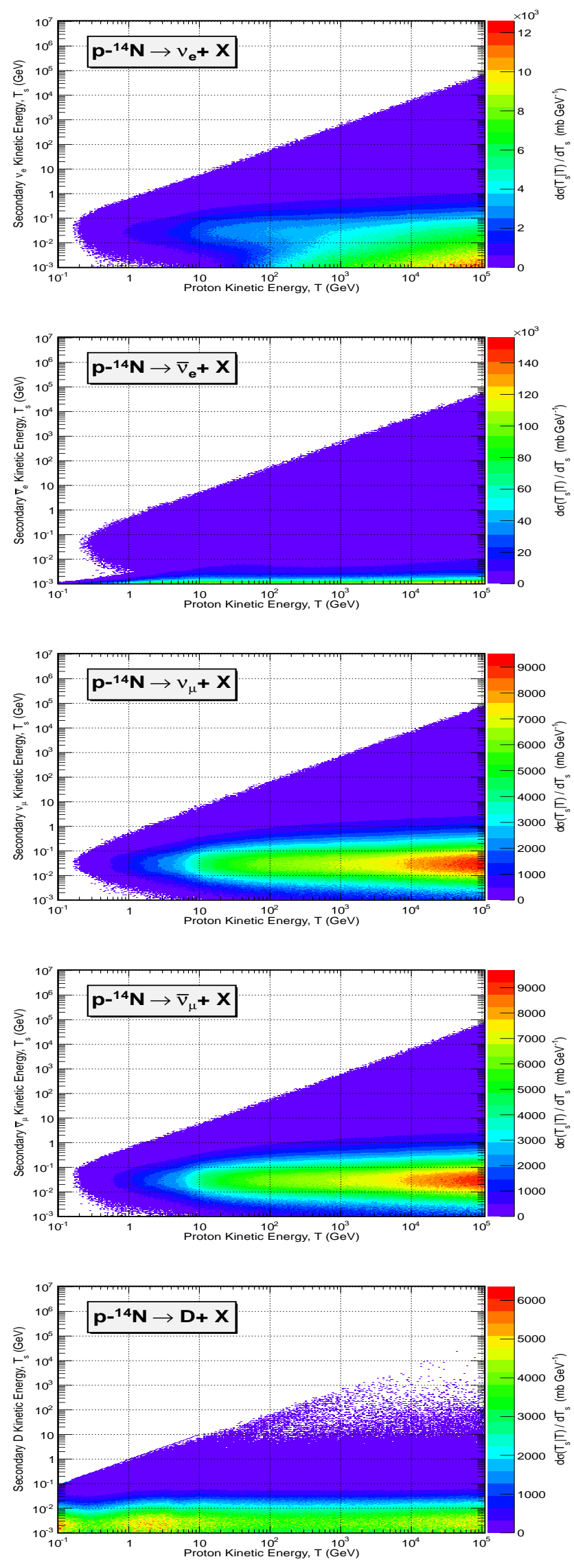
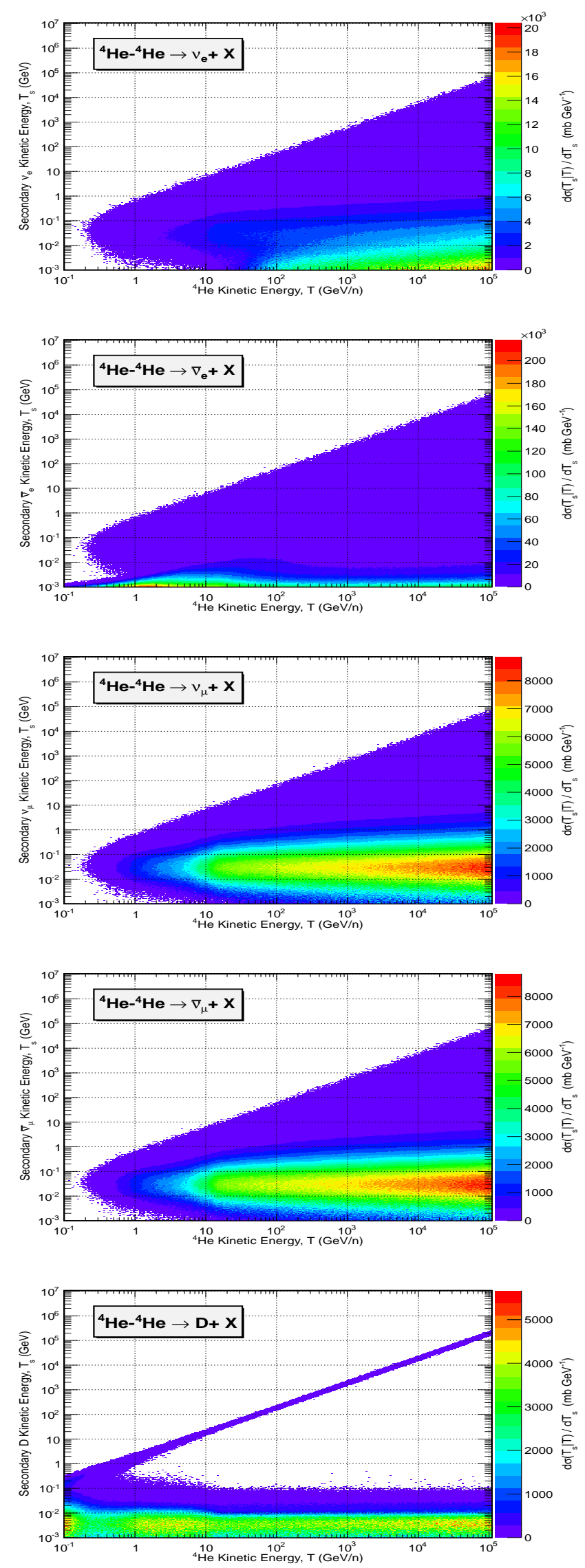

Figure A.15: Differential inclusive secondary cross sections for the production of $v, \bar{v}$ and deuteron in $p-{ }^{14} N$ (left) and ${ }^{4} H e-{ }^{4} H e$ (right) interactions. The values of the cross sections (color scales) are in $\mathrm{mb} \mathrm{GeV}^{-1}$. 


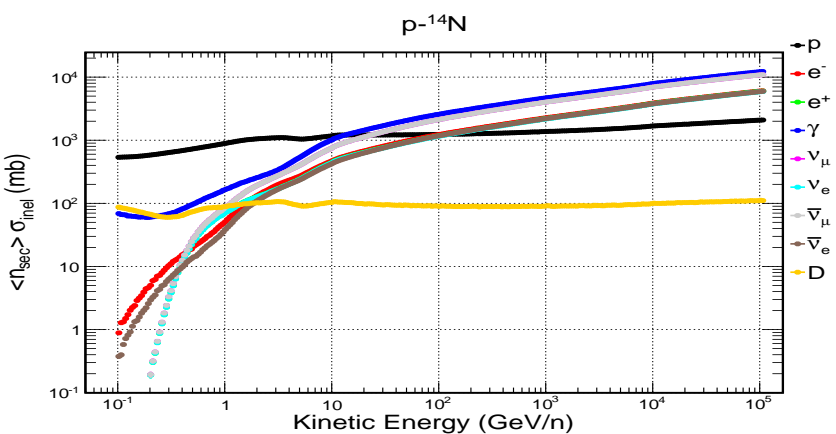

p- ${ }^{40} \mathrm{Ar}$
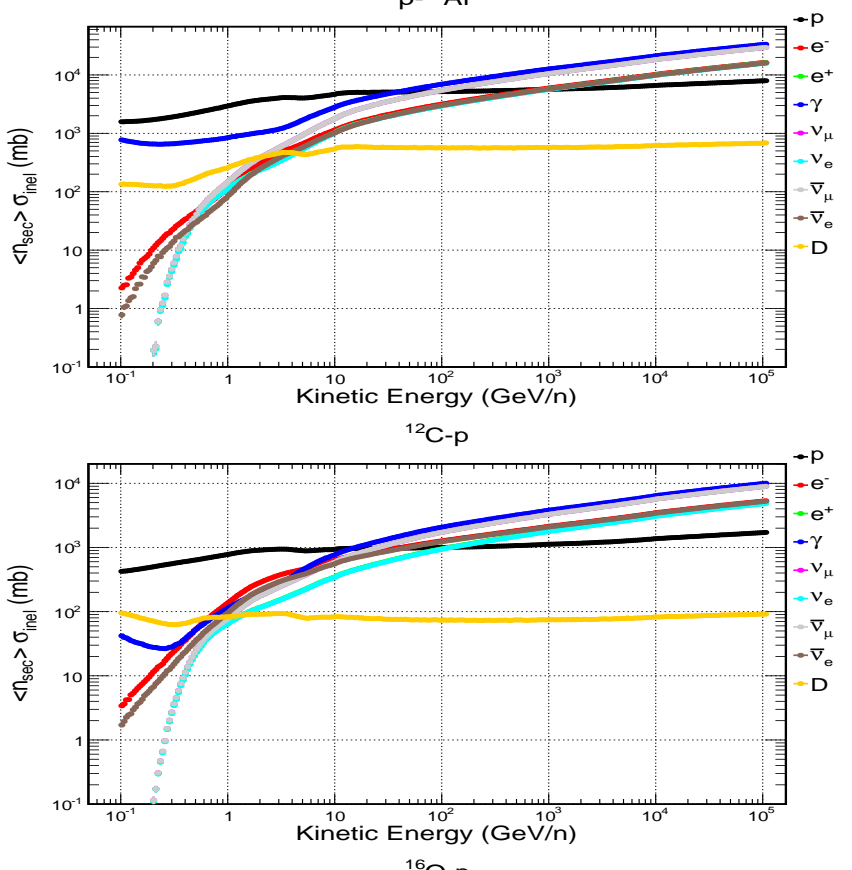

${ }^{16} \mathrm{O}-\mathrm{p}$
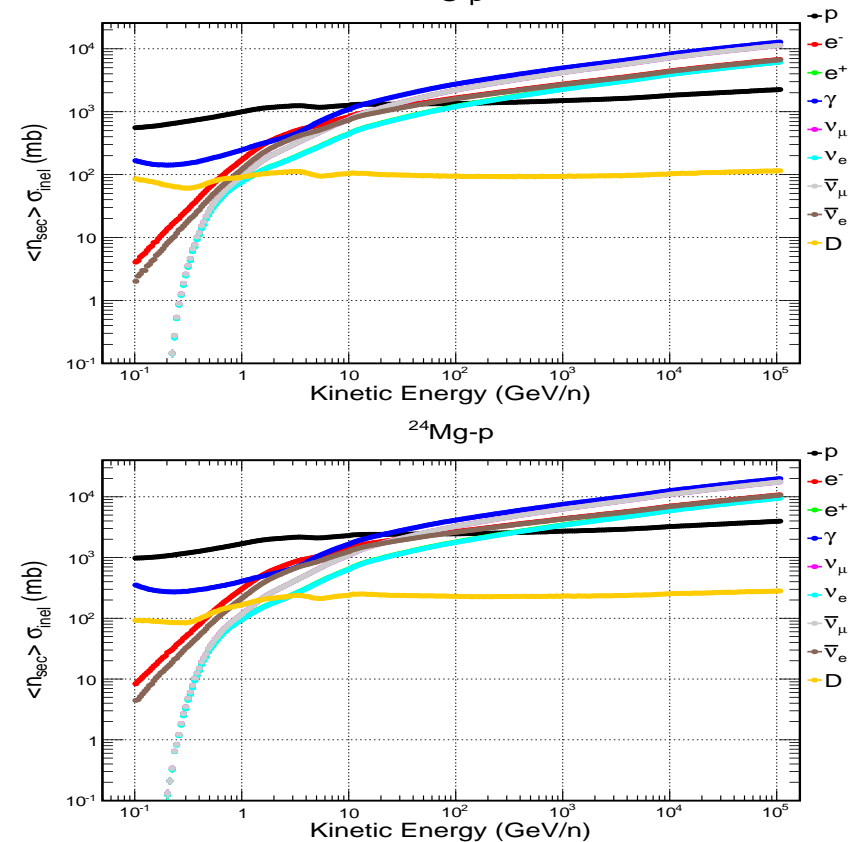

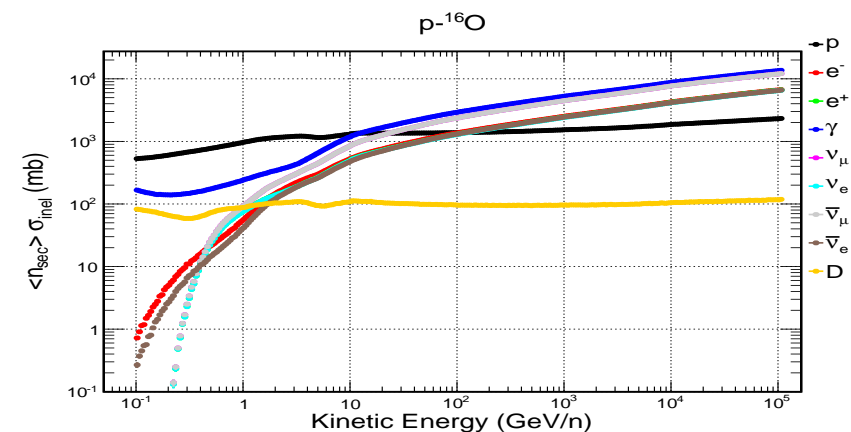

D-p

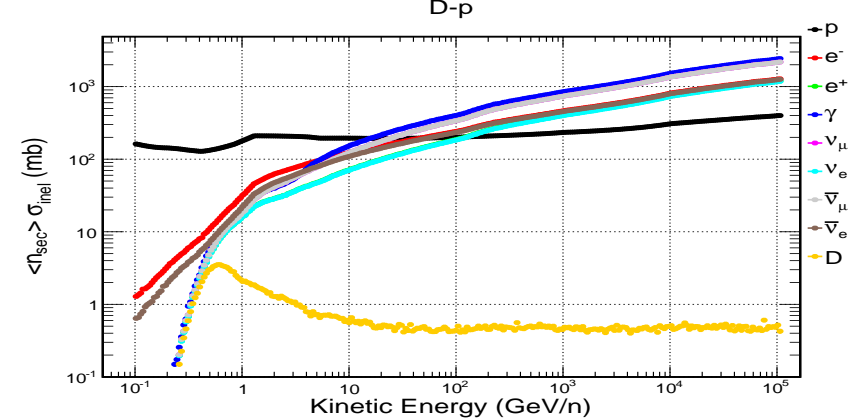

${ }^{14} \mathrm{~N}-\mathrm{p}$

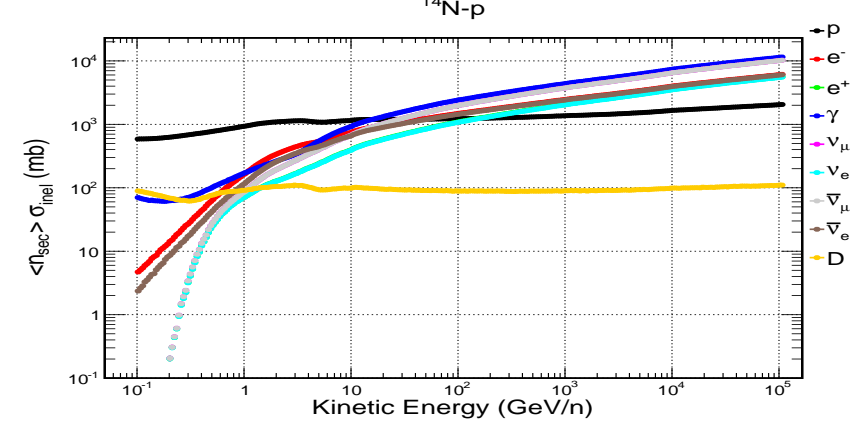

${ }^{20} \mathrm{Ne}-\mathrm{p}$
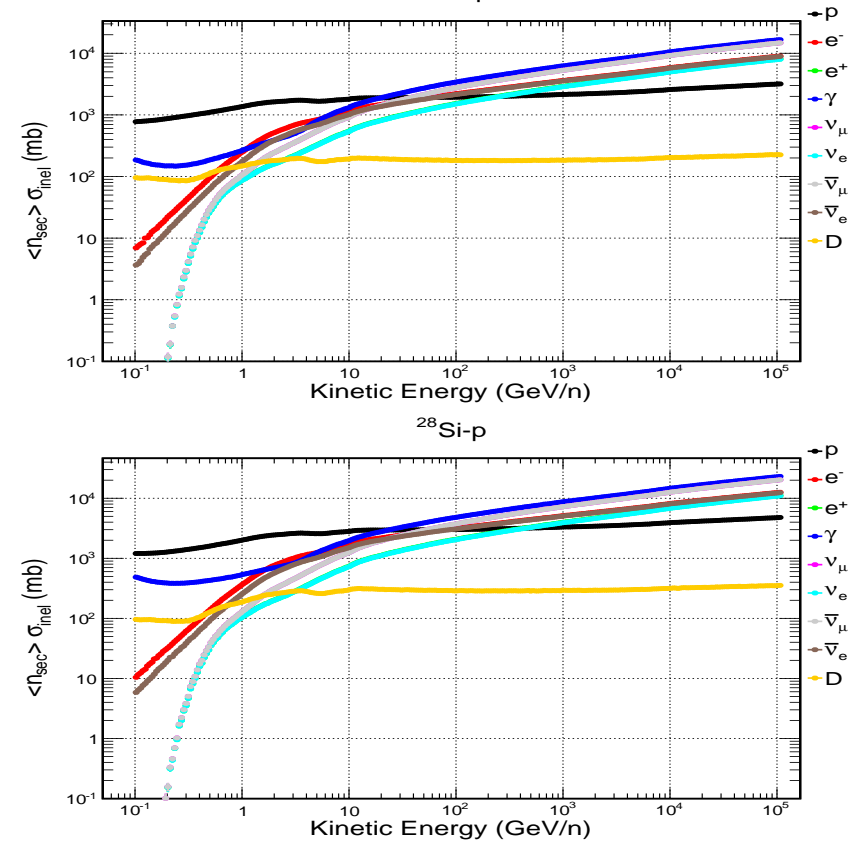

Figure A.16: Inclusive cross sections for the production of protons (black), electrons (red), positrons (green), gamma rays (blue), electron neutrinos (cyan), electron antineutrinos (grey), muon neutrinos (magenta), muon antineutrinos (brown) and Deuterons (orange) in the collisions of several CR projectiles with different target nuclei. 

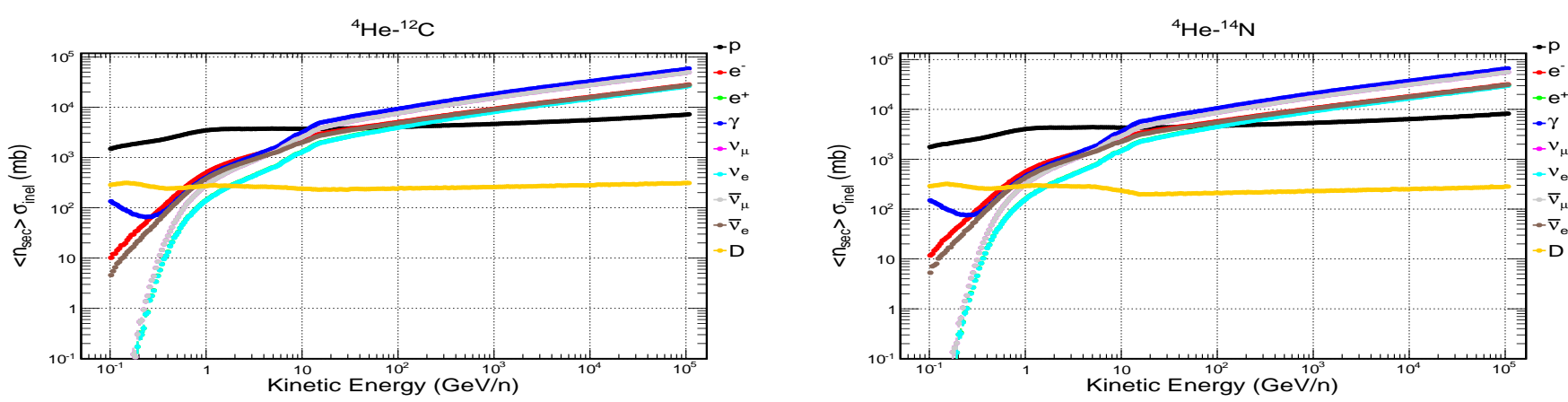

${ }^{4} \mathrm{He}-{ }^{16} \mathrm{O}$
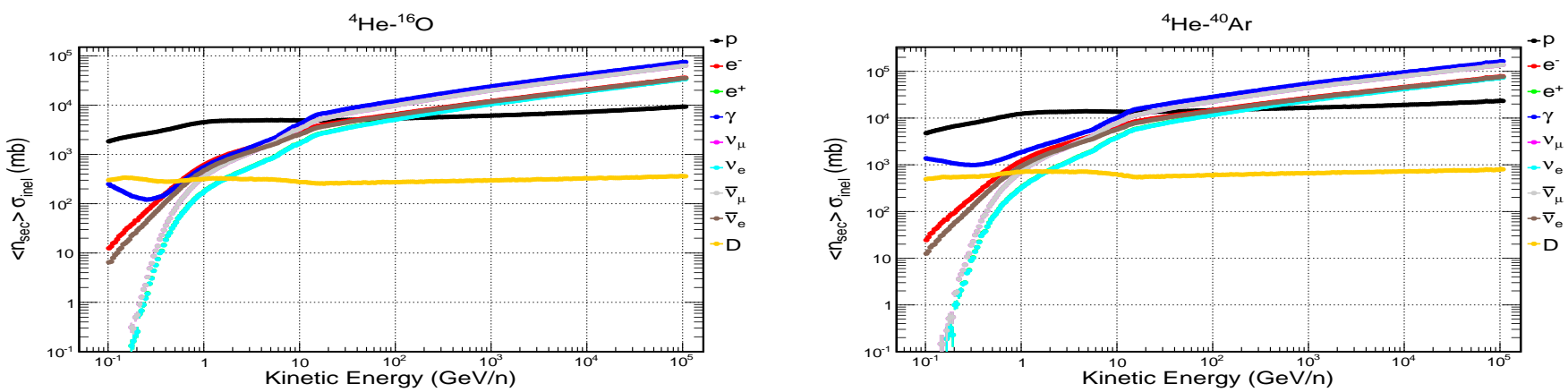

${ }^{12} \mathrm{C}-{ }^{4} \mathrm{He}$
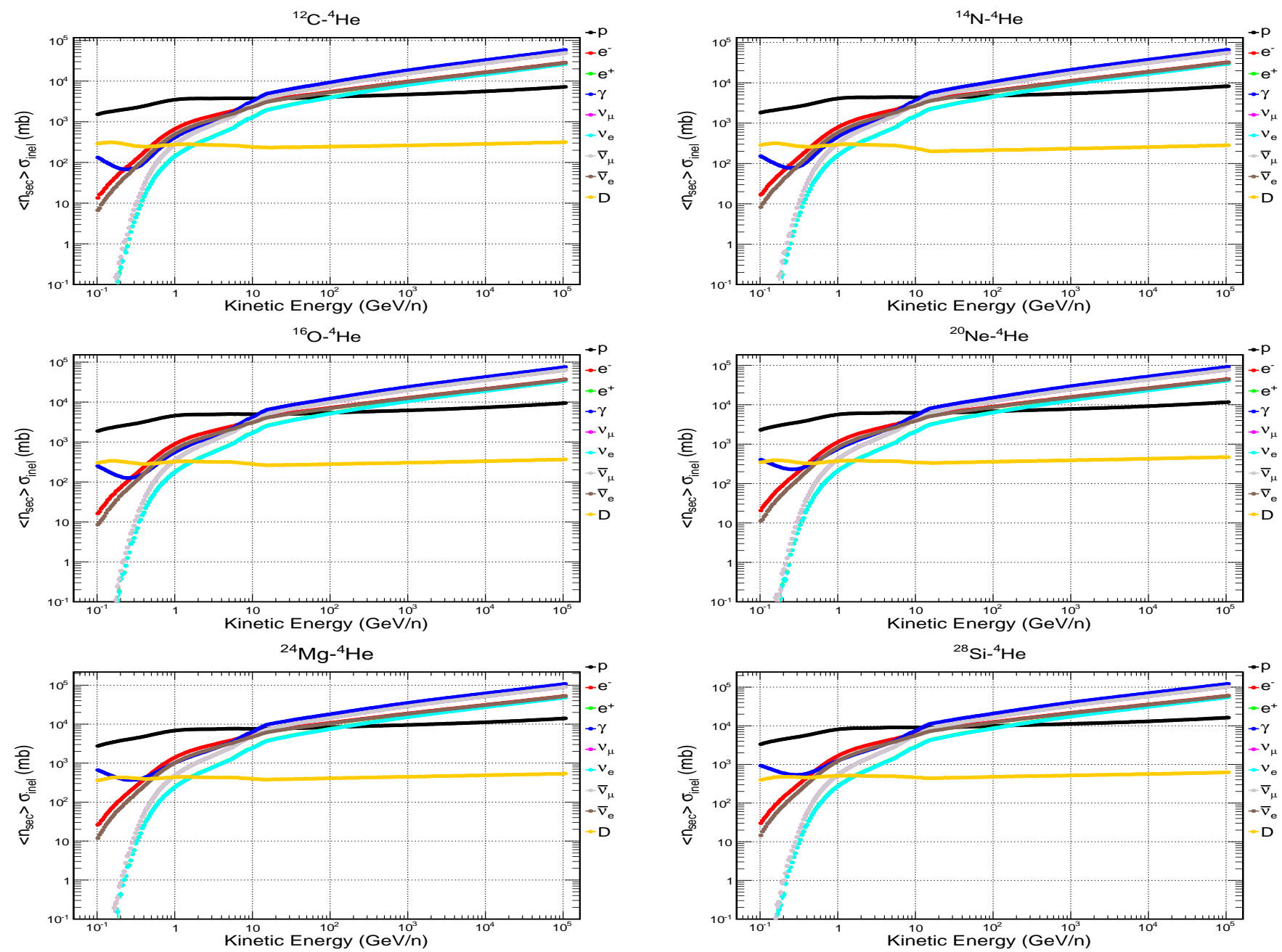

Figure A.17: Inclusive cross sections for the production of protons (black), electrons (red), positrons (green), gamma rays (blue), electron neutrinos (cyan), electron antineutrinos (grey), muon neutrinos (magenta), muon antineutrinos (brown) and Deuterons (orange) in the collisions of several CR projectiles with different target nuclei. 

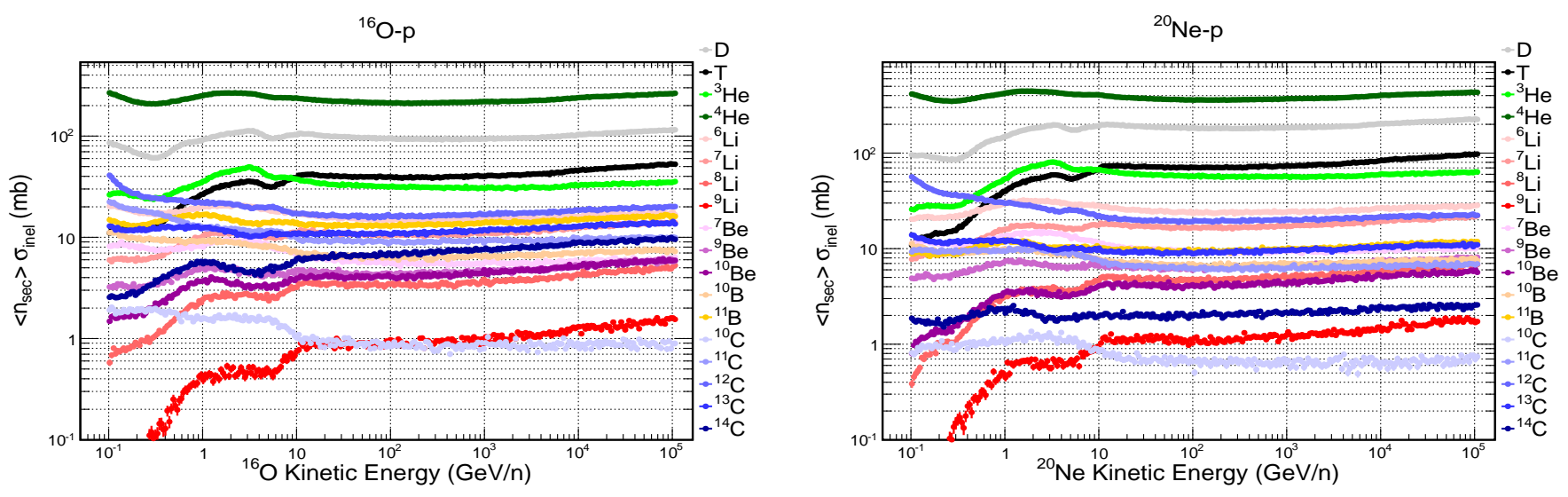

${ }^{24} \mathrm{Mg}-\mathrm{p}$
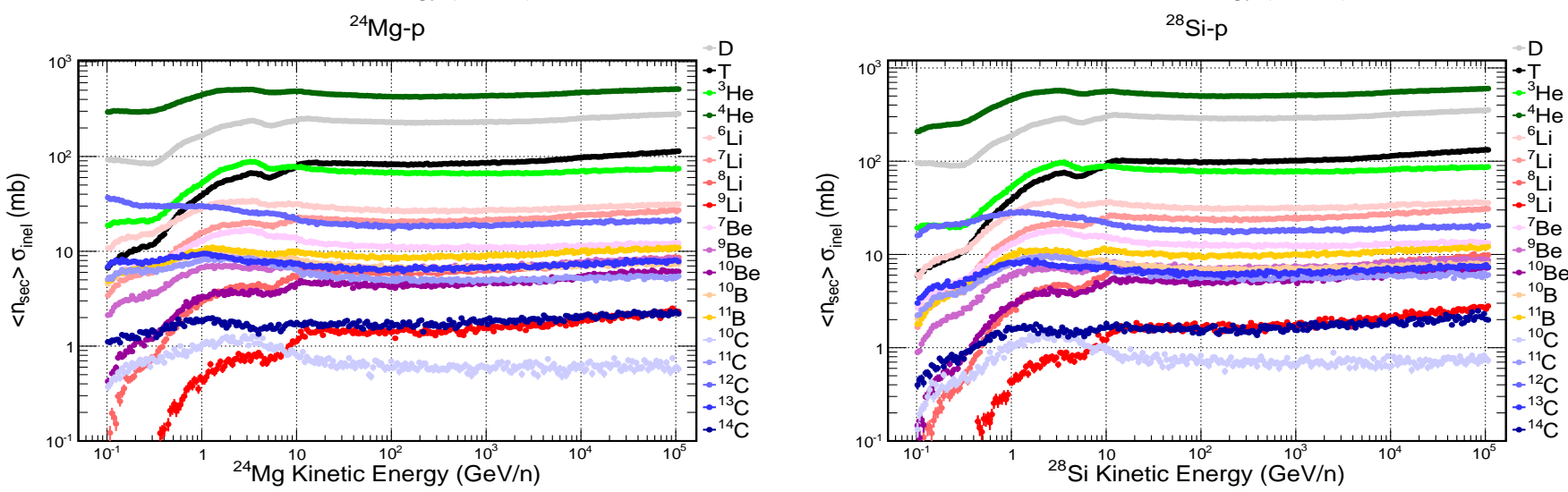

${ }^{16} \mathrm{O}-{ }^{4} \mathrm{He}$
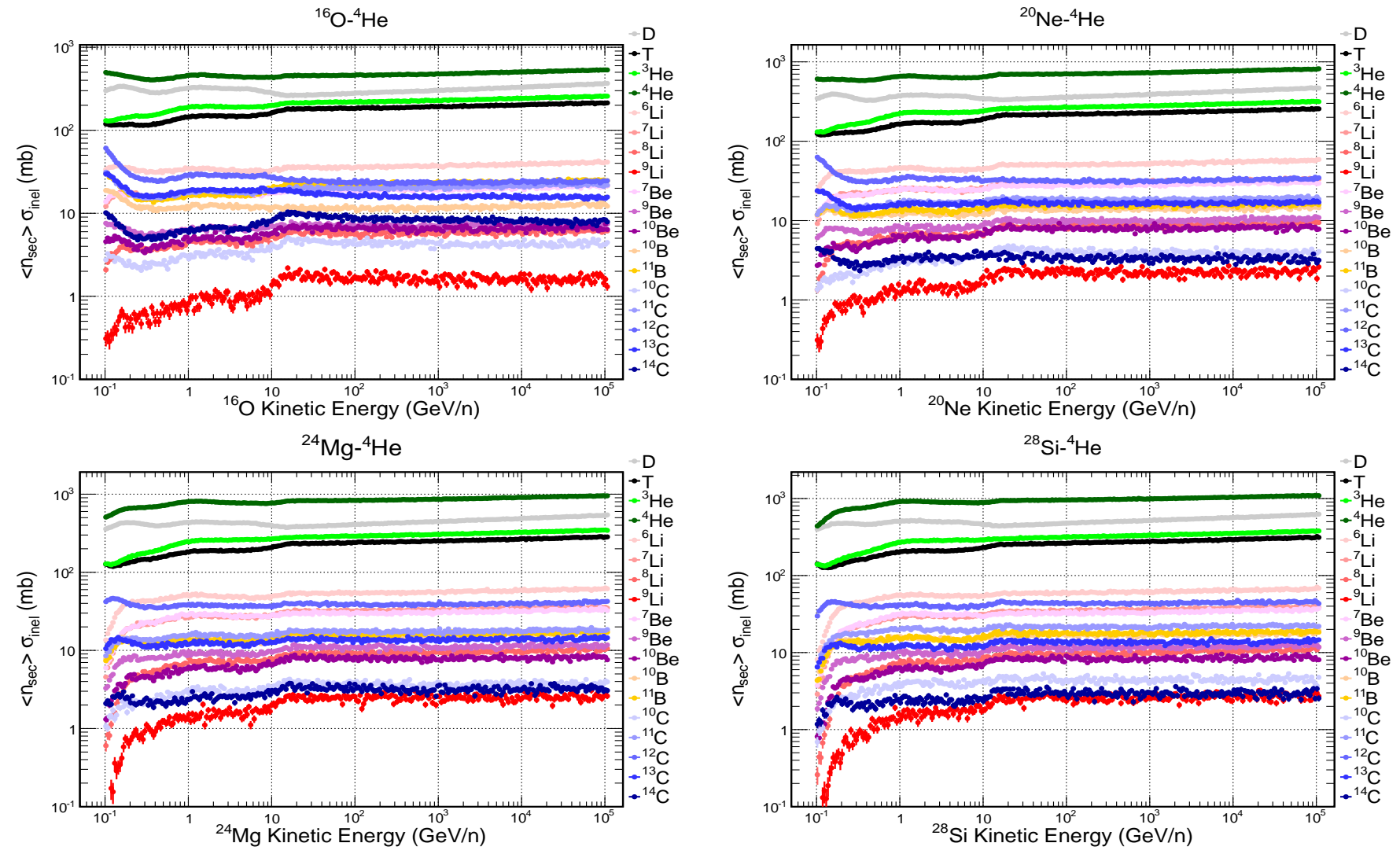

Figure A.18: Inclusive cross sections for the production of spallation nuclei in collisions of ${ }^{16} \mathrm{O},{ }^{20} \mathrm{Ne},{ }^{24} \mathrm{Mg}$, and ${ }^{28} \mathrm{Si}$ with p and ${ }^{4} \mathrm{He}$ nuclei. The plots show the cross sections for the production of Deuteron (gray markers), Triton (black markers) and for the isotopes of $\mathrm{He}\left({ }^{3} \mathrm{He}\right.$ and ${ }^{4} \mathrm{He}$, green markers), $\mathrm{Li}\left({ }^{6} \mathrm{Li},{ }^{7} \mathrm{Li},{ }^{8} \mathrm{Li}\right.$ and ${ }^{9} \mathrm{Li}$, red markers), $\mathrm{Be}\left({ }^{7} \mathrm{Be}\right.$ and ${ }^{9} \mathrm{Be}$, magenta markers), $B\left({ }^{10} \mathrm{~B}\right.$ and ${ }^{11} \mathrm{~B}$, orange markers) and $C\left({ }^{10} \mathrm{C},{ }^{11} \mathrm{C},{ }^{12} \mathrm{C},{ }^{13} \mathrm{C}\right.$ and ${ }^{14} \mathrm{C}$, blue markers). Lighter (darker) color shades correspond to lighter (heavier) isotopes. 